\title{
Densidad de siembra y comportamiento agronómico de tres variedades de maíz morado (Zea mayz L.)
}

\author{
Mario Pedraza Guevara ${ }^{1}$, Guillermo Idrogo Vazquez ${ }^{2}$ y Santos Pedraza Guevara ${ }^{3}$ \\ ${ }^{1}$ Universidad Nacional Toribio Rodríguez de Mendoza de Amazonas (UNTRM), Chachapoyas, Amazonas \\ Perú \\ ${ }^{2}$ Universidad Nacional Toribio Rodríguez de Mendoza de Amazonas (UNTRM), Chachapoyas, Amazonas \\ Perú \\ ${ }^{3}$ Universidade Tecnológica Federal de Paraná (UTFPR), Ponta Grossa, Paraná, Brasil
}

Recibido el 16 de junio del 2017. Aceptado el 20 de julio del 2017

DOI: https://doi.org/10.33017/RevECIPeru2017.0003/

\section{Resumen}

El objetivo fue evaluar el efecto de tres densidades $[40 \mathrm{~cm} \times 60 \mathrm{~cm}, 30 \mathrm{~cm} \times 50 \mathrm{~cm}$ y $20 \mathrm{~cm} \times 60 \mathrm{~cm}$ ] de siembra en el comportamiento agronómico de tres variedades de maíz morado (Zea mayz L.) INIA-601, UNC-47 y PMV_ 581. Para ello se realizaron muestreo de suelo. Las labores culturales comenzaron con la preparación del terreno, siembra, manejo del cultivo y cosecha. El proyecto se localizó en el anexo Quipachacha, distrito de Levanto región Amazonas a 2820 msnm, Estación Experimental UNTRM, INDES- CES. El área de terreno fue de $844-8 \mathrm{~m}^{2}$ con dimensiones de $13,20 \mathrm{~m}$ ancho por $64 \mathrm{~m}$ de largo. El diseño estadístico fue factorial de 3 bloques (repeticiones) y 09 parcelas demostrativas (tratamientos), con separación de $1_{-5} 0 \mathrm{~m}$ entre parcelas y $1.5 \mathrm{~m}$ entre bloques, además de 1 testigo $60 \mathrm{~cm} \times 40 \mathrm{~cm}$. Para la contrastación de la hipótesis $(\mathrm{p}<0.05)$ se utilizó el análisis de variancia de los factores y variables de estudio y su respectiva prueba de Tukey en SPSS (Versión 23.0). El mayor porcentaje de germinación de 96.80 se encontró en la variedad de maíz morado UNC - 47 a densidad de siembra de $1.5 \mathrm{~m}^{2}$. A los 93 días se desarrolló la floración sin diferencia significativa entre tratamientos. La variedad INIA - 601 sobresalió en mayor altura de planta $202.6 \mathrm{~cm}$ (tratamiento 1) a densidad $60 \mathrm{~cm} \times 40$. Asimismo, 04 plantas/m lineal, 1.43 mazorca/planta a densidad $50 \mathrm{~cm} \times 30$ y mayor peso de semillas $5.497 \mathrm{~g}$. Del mismo modo, la variedad PVM - 581 sobresalió con $145 \mathrm{~cm}$ de inserción de mazorca, es decir, a densidad de $60 \mathrm{~cm} \times 20 \mathrm{~cm}$. El mayor número de hileras por mazorca fue 11.83 y longitud de mazorca $20.76 \mathrm{~cm}$ a densidad de $60 \mathrm{~cm} \times 40 \mathrm{~cm}$. El mayor diámetro de mazorca se encontró en la variedad PVM - $581(4.93 \mathrm{~cm})$ a densidad de $50 \mathrm{~cm} \times 30 \mathrm{~cm}$. Se encontró un rendimiento promedio de maíz morado de $4808.80 \mathrm{~kg} / \mathrm{ha}$ en la variedad INIA - 601 a densidad $60 \mathrm{~cm} \times 40 \mathrm{~cm}$, por consiguiente menor diámetro de mazorca. Los menores rendimiento se encontraron en PVM - 581 a densidades $2745.50 \mathrm{~kg} / \mathrm{ha}$.

DescriptoresPalabras-claves: densidad de siembra, comportamiento agronómico, maíz morado, zea mayz

\section{Abstract}

The aim of this study was to evaluate the effect of three densities $[40 \mathrm{~cm} \times 60 \mathrm{~cm}, 30 \mathrm{~cm} \times 50 \mathrm{~cm}$ and $20 \mathrm{~cm} \times 60 \mathrm{~cm}$ ] on the agronomic behavior of three varieties of purple maize (Zea mayz L.) INIA-601, UNC-47 and PMV-581. Thus, soil sampling was carried out. The cultural work began with the preparation of the land, planting, handle of the crop and harvest. The project was located in the Quipachacha annex, Levanto district in the region of Amazonas at $2820 \mathrm{msnm}$, UNTRM Experimental Station, INDES - CES. The area of land was $844.8 \mathrm{~m}^{2}$ with dimensions of $13.20 \mathrm{~m}$ wide by $64 \mathrm{~m}$ long. The statistical design was factorial of 3 blocks (repetitions) and 09 demonstration plots (treatments), with separation of $1.0 \mathrm{~m}$ between plots and $1.5 \mathrm{~m}$ between blocks, furthermore, 1 control $60 \mathrm{~cm} \times 40 \mathrm{~cm}$. The analysis of variance of the factors and study variables and their respective Tukey test in SPSS (Version 23.0) were used to test the hypothesis $(p<0.05)$. The highest percentage of germination 
of 96.80 was found in the variety of UNC - 47 purple maize at planting density of $1.5 \mathrm{~m}^{2}$. At 93 days flowering was developed without significant difference between treatments. The variety INIA - 601 stood out at a higher plant height of $202.6 \mathrm{~cm}$ (treatment 1 ) at $60 \mathrm{~cm} \times 40$ density. Moreover, 04 plants/linear $\mathrm{m}, 1.43 \mathrm{cob} /$ plant at density $50 \mathrm{~cm} \times 30$ and greater weight of seeds $5,497 \mathrm{~g}$. In the same way the variety PVM - 581 excelled with $145 \mathrm{~cm}$ of insertion of corn that is to say to density of $60 \mathrm{~cm} \times 20 \mathrm{~cm}$. The highest number of rows per ear was 11.83 and ear length $20.76 \mathrm{~cm}$ at density of $60 \mathrm{~cm} \times 40 \mathrm{~cm}$. The diameter[M1]> of cob was found in the variety PVM -581 (4.93 $\mathrm{cm}$ ) at density of $50 \mathrm{~cm} \times 30 \mathrm{~cm}$. An average yield of purple maize of $4808.80 \mathrm{~kg} / \mathrm{ha}$ was found in the variety INIA - 601 at density $60 \mathrm{~cm} \times 40 \mathrm{~cm}$, consequently lower diameter of ear. The lowest yields were found in PVM - 581 at densities $2745.50 \mathrm{~kg} / \mathrm{ha}$.

\section{Keywords: planting density, agronomic performance, purple corn, zea mays}

\section{Introducción}

En maíces, existen muchas variedades desde los colores, blanco, negro, amarillo, morado, colorado claro, y oscuro, y mezclado de varios colores [4]. El maíz morado originalmente se cultiva desde hace siglos en la región andina de América del Sur principalmente Perú, Bolivia [5], Ecuador [2, 3] y México [6]. Como alimento en el Perú Zea mays habita en las vertientes occidentales del Perú alrededor de $8^{\circ} 00^{\prime} 28,8^{\prime \prime}$ S, 7841'18,5" W y 1390 msnm. Sobre su conocimiento popular en la población nor - costeña peruana (región La Libertad), utilizaban como alimento desde épocas prehispánicas. Asimismo, se identifican y describen representaciones del mencionado cereal en la colección de cerámica prehispánica que custodia el Museo de Historia Natural y Cultural de la Universidad Privada Antenor Orrego de Trujillo, Perú. Se han encontrado restos con una antigüedad mayor a 4000 años: Cuevas de Guitarrero en la sierra de Ancash (6000 a 8000 años), las Haldas (Casma) hay evidencias de su consumo años 1500 a.c. [7] en cuevas de Rosamachay en Ayacucho (5500 años), Los Gavilanes y Aspero en Huarmey (4500 años). En los Gavilanes, por ejemplo, se han identificado variedades Confite Chavinense, Proto Confite Morocho y Proto Kcully, que se cultivaban en la costa peruana hace más de 4000 años según Grobman (1982) citado por [8]; seguidamente se introdujo en China [9].

Estudios de maíz morado, revelaron la presencia de algunas especies ancestrales primitivas como el Kulli e incipiente el Morado Canteño. Su cultivo se realiza principalmente en regiones de Cajamarca, Ayacucho, Ancash, Lima y Arequipa [6]; obtienen semillas de diversas estaciones experimentales del INIA (Instituto Nacional de Innovación Agraria) (INIA) con poca frecuencia de mercados informales como "la Parada" en Lima, mercados de la localidad. Otros optan por experimentar con semillas obtenidas de "Asociaciones de productores de semillas de la Universidad Nacional Agraria La Molina (UNALM)" [10].

Sin embargo, el Programa Nacional de Innovación Agraria en Maíz del INIA, ha dispuesto una nueva variedad INIA - 601 obtenida mediante polinización abierta tras largo perílodo de investigación en Cajamarca. Dada las condiciones óptimas de temperatura en Quipachacha - Levanto Región Amazonas del Perú (2820 msnm) de siembra además estas variedades INIA - 47 y PVM - 581 . Los objetivos del proyecto de la tesis[M2] fue (i) evaluar el efecto de tres densidades de siembra en el comportamiento agronómico en tres variedades de maíz morado (Zea mayz L.) INIA - 601, UNC 47 y PMV - 581 variedad clasificada en Perú por el INIA [1]; así como identificar la mejor densidad de siembra entre surcos y plantas de las variedades en evaluación, (iii) determinar parámetros agronómicos y rendimiento.

\section{Marco teórico}

\subsection{Caracterización botánica del maíz}

Según Manrique (1997), el maíz (Zea mays L.) es una gramínea con un gran desarrollo vegetativo que puede alcanzar hasta $5 \mathrm{~m}$-Mt de altura.

Taxonomía: de nombre común maíz morado pertenece al reino Plantae, Monocotiledóneas, de la familia de las Gramineae o Poaceae [2, 3]. En algunos lugares y regiones es conocido como maíz en español, milho en portugués, sara en Quechua, tonqo en Aymara en el Perú.

\subsection{Importancia, uso y subproductos del maíz morado}

El maíz morado tiene mucha importancia y relevancia en la industria porque poseer una cantidad considerable de antioxidantes, $6 \mathrm{~g} / \mathrm{kg}$ de antocianinas [9, 11]. El colorante extraído de maíz morado (Zea mays L.) fue utilizado por la civilización 
Inca para preparar alimentos y como colorante natural tiñendo fibras textiles [12]. Actualmente en el Perú, se consume como bebida típica comúnmente llamada "chicha morada" y postres (mazamorra) en la industria se usa como colorante [13] y para mejorar la salud [14, 15] "colada morada" en Ecuador ancestral y sincretismo religioso en finados [2]. Otras zonas productoras del maíz morado se consumen en preparados de tamales y humitas de coloración morada. En Ayacucho se ha preparado chicha de jora de coloración similar al vino (INIA, 2016). Es generador de mejores ingresos económicos, principalmente, para agricultores de los valles interandinos de la sierra peruana (INIA, 2012). El colorante del maíz morado es de color púrpura oscuro a casi negro, se debe al alto contenido de antocianinas (pigmento azul morado) tales como cianidina-3-glucósido, pelargonidin-3glucósido, y peonidin-3-glucósido [16, 17]. Este pigmento es utilizado como aditivo natural en la coloración en la industria de alimentos y bebidas [14], productos lácteos, productos de panadería, productos vegetales, conservas de pescado, grasas, aceites, mermeladas, jaleas, frutas confitadas, frutas en almíbar, jarabes de frutas, sopas, almíbar; también es usado como tinte en tejidos y en la industria de cosméticos [11]. Por sus cualidades medicinales es utilizado en la industria farmacéutica [14]. El grano se puede aprovechar para la extracción de almidones y/o derivados o en la elaboración de alimentos balanceados para animales [2]. Su uso previene enfermedades degenerativas como la arteriosclerosis, diabetes y artritis. Reduce la presión arterial y colesterol en la sangre $[9,11]$.

\subsection{Producción en la región Amazonas}

Existe producción de maíz común (amarillo, amiláceo); que se venden en plazas y/o mercados. Se necesita implementar nuevas alternativas de producción, mediante la introducción de otras variedades, como el maíz morado que conlleve a diversificar los cultivos, coadyuvando así a los agricultores a elevar sus ingresos económicos por ser variedades que contiene pigmentos antonianos. Del mismo modo, hace falta la capacitación al agricultor. Según el IV CENAGRO (2012), la superficie promedio de cultivo de maíz morado a nivel nacional y especialmente la región Amazonas es 1,2000/ha, con 04 unidades agropecuarias (PERU INEI - IV Censo Nacional Agropecuario 2012). A nivel nacional la producción de UA de superficie promedio por provincia. La mayor producción se encuentra en la Provincia de Bongará $(0,5)$, seguido de Chachapoyas $(0,2)$, Luya $(0,25)$.
Esto se debe a los climas y pisos ecológicos aptos para la adaptación del cultivo de maíz morado. No se registró datos de producción en el Distrito de Levanto. En cambio sí para la provincia de Lima con registros de producción de maíz morado 2.314,2309 en superficie y de 1.626 UA, seguido de Ancash con una superficie de cultivo de 1.490,2131 y una producción de 1.371 UA. Ayacucho ocupa el cuarto lugar después de Arequipa y finalmente Cajamarca registra datos de producción 910 de UA (IV CENAGRO, 2012).

\subsection{Mercado del maíz morado}

Perú exporta maíz morado a más de 13 países de tres continentes, donde es usado como fármaco por sus efectos: Alemania, México, EE.UU. y Vietnam; en China y Turquía, la vaina o cubierta de grano es utilizada para el tratamiento de diarreas y el grano entero en metrorragias; asimismo, es utilizado como antidiabético, hipotensor, digestivo. En Cuba y Haití, las semillas molidas, son utilizadas como cataplasmas para golpes, torceduras y fracturas; en Filipinas para el tratamiento de edemas de las embarazadas; en Taiwán para el tratamiento de la Hepatitis; en Trinidad y Tobago, las semillas son usadas como antidiarréicas, analgésicas antisépticas y diuréticas [3, 18]. Por su gran valor en presencia de pigmentos (antocianinas) el maíz morado en el 2006, el Perú exportó 338,1 TM en diferentes presentaciones (entero 29\%), coronta (28\%), extracto, colorante, congelado, harina, seco, capsulas, granos, concentrados, jugo, extracto y presentaciones no específicas el $17 \%$ por un valor de US\$633,3 mil, lo que significó un crecimiento en $11,6 \%$, en valor y $2,1 \%$ en volumen, respecto al 2005, atribuido al incremento de población latina, especialmente de origen peruana, en el exterior, lo que determina variaciones en los hábitos de consumo (MINAGRI, 2014, Solid Perú). Los principales mercados de destino del maíz morado entero y sus diferentes presentaciones son: EE.UU (55\%) y Japón (31\% principalmente de coronta). El 2007 la exportación de maíz morado ascendió a US\$ 386 690, siendo Estados Unidos con el $60 \%$ el principal mercado, seguido por Japón, España e Italia [19].

En los diez primeros meses del 2008, 15 países importaron maíz morado; EE.UU lo hizo por un valor de US\$159.960 que representó el $56 \%$ del total de producto comercializado internacionalmente, Japón compró US\$ 88.58 en maíz morado (31\%), España US\$23.703 (8,3\%) e Italia US\$ 6.364 (2,2\%). Otros países importadores en menores cantidades fueron Canadá, China, Venezuela, Costa Rica, Chile y 
Alemania, que en conjunto compraron US\$3.745. En EE.UU, los comerciantes hispanos de Nueva York formaron una asociación que agrupa a más de 7.000 bodegas que buscan abastecimiento para su marca "Mi Bodega"; el maíz morado es distribuido por esa cadena a la que proveen exportadores peruanos (MINAGRI, 2016).

\subsection{Empresas exportadoras}

El año 2006, las empresas con mayores exportaciones de maíz morado fueron San El-Gen FFI Perú (27,2\% del total exportado), Montana $(10,5 \%)$ y The Green Farmer (10,2\%) con un precio de S/. 3.6 (nuevos soles) [46].

\subsection{Normas de producción de maíz morado en el Perú}

El maíz morado se encuentra dentro de las 45 especies nativas cultivadas y silvestres con la ley 28477, protege al maíz morado como "Patrimonio Natural de la Nación".

Tal que las exigencias de cumplimiento de los mercados mundiales en cuanto a producción de calidad, ha hecho que el Perú obtenga la certificación KOSHER. Por lo que se está exportando maíz morado con características físicas de 12 a 14\% de humedad, libre de enfermedades y envasado. El Código E-163, según el anexo № 02. Código para la unión europea y japonesa. Las características del producto 100\% natural, con certificación de KOSHER lo que implica mayores ventajas del consumo del maíz morado y sus derivados, asimismo, incentivar la promoción (El Peruano, diario oficial - Normas Legales 351683, agosto 2007; PETT, Shama 2004 y Solid Perú).

El rendimiento de la coronta va a depender de acuerdo a las variedades se estima que representa entre el $16 \%$ al $20 \%$ del rendimiento de mazorcas, utilizando semilla mejorada y buen manejo agronómico se obtiene de 1200 a $1600 \mathrm{~kg} / \mathrm{ha}$ de coronta. El precio de coronta en el mercado de exportación, en 2006 estuvo en promedio a 3.24 de dólares por kilogramo, lo que significa que se debe obtener ingresos de 3500 a 5000 dólares/ha por venta de coronta de maíz morado. Coronta molida es otra forma de presentación que los agricultores deben implementar en forma organizada para obtener mejores ingresos económicos.

\section{Cuadro[M3] 1 - Descripción de producción a nivel tecnológico en maíz morado}

\begin{tabular}{lll}
\hline Detalle & Tecnología media & Tecnología baja \\
\hline Cultivo & Maíz morado & Maíz morado \\
Variedad & Morado Canteño & Morado Canteño \\
Periodo vegetativo & $5-6$ meses & 6 meses \\
Distanciamiento de siembra & $0.8 \times 0.45 \mathrm{~m}$ & $0.8 \times 0.45 \mathrm{~m}$ \\
Rendimiento por (kg/ha) & 3800 a 4000 & 1400 a 2000 \\
Jornal / Día (S/.)(promedio) & 12.5 & 12.5 \\
Tractor /Yunta Hora (S/.) & 45 & 45 o yunta (S/. 40) \\
Fertilización/Dosis/ha) & $180-80-60$ & $90-50-60$ \\
Densidad de siembra (Plantas/ha) & 82,000 & Al boleo \\
Época de siembra & Agosto a octubre & Agosto a octubre \\
\hline
\end{tabular}

Fuente: Lazo R., 1999; Zevallos M., 1999 \& Puma J. 1998.

\subsection{Distribución geográfica, requerimientos climáticos:}

El maíz es uno de los cuatro cultivos más importantes del mundo, por la cantidad de has cultivadas y su aporte a la alimentación (FAO, 2016). Debido a las múltiples variedades, esta gramínea puede aclimatar desde el nivel del mar hasta los $3500 \mathrm{msnm}$ con mejores rendimientos [20].

2.7.1. Suelo: El maíz se adapta a suelos de textura franca, franco-arcilloso y franco-limoso, con pH de 6.5 a 7.5 es donde se aprecia el mejor desarrollo. Requieren además suelos profundos, ricos en materia orgánica con buen drenaje [21]. El comportamiento de crecimiento del maíz se ve influenciado por la permeabilidad del perfil del suelo, tiene correlación positiva significativas con la porosidad total del suelo, porosidad no capilar, contenido inicial de humedad, capacidad de retención de agua, contenido de materia orgánica y compuestos NPK, así como del genotipo o variedad [22], y la correlación lineal negativa significativa con la densidad aparente del suelo [16]. La densidad de siembra depende de la fertilidad del suelo y del objetivo de estudio [20, 23]. Asimismo la aparición de malas hiervas en el cultivo maíz causa pérdidas que limitan el rendimiento de grano [24]. 
2.7.2. Clima: El maíz suave se cultiva entre los 2200 a 3100 msnm en un clima templado frío y sub-cálido, requiere de una temperatura de 10 a $20^{\circ} \mathrm{C}$. Es importante que la temperatura óptima para la germinación de la semilla esté entre los 15 a $20^{\circ} \mathrm{C}$. [21]. La FAO (2012), señala que el maíz requiere una temperatura entre 15 y $30^{\circ} \mathrm{C}$, puede soportar temperaturas mínimas de $8^{\circ} \mathrm{C}$ y máximas de $30^{\circ} \mathrm{C}$; pero la temperatura ideal para el desarrollo de la mazorca debe estar entre los 20 a $32^{\circ} \mathrm{C}$. El maíz requiere de un porcentaje de humedad que está entre $80-90 \%$, una pluviosidad que va desde los 700 a $1300 \mathrm{~mm}$ (FAOSTAT, 2016), el bajo rendimiento puede deberse al estrés hídrico que vaya a sufrir la planta [25]. Cada etapa fenológica tiene un requerimiento en particular, conforme se señala en la Tabla 1.

Tabla 1: Requerimientos térmicos según ciclo fenológico del maíz

\begin{tabular}{llll}
\hline $\begin{array}{c}\text { Proceso } \\
\text { fisiológico }\end{array}$ & & Temperatura $\left({ }^{\circ} \mathrm{C}\right)$ \\
\hline Curso & Mínima & Óptima & Máxima \\
\hline Germinación & 10 & $20-25$ & 40 \\
Crecimiento & 15 & $20-30$ & 40 \\
Floración & 20 & $21-30$ & 30
\end{tabular}

Fuente: FAOSTAT Date: Fri Sep 16 19:56:10 CEST 2016

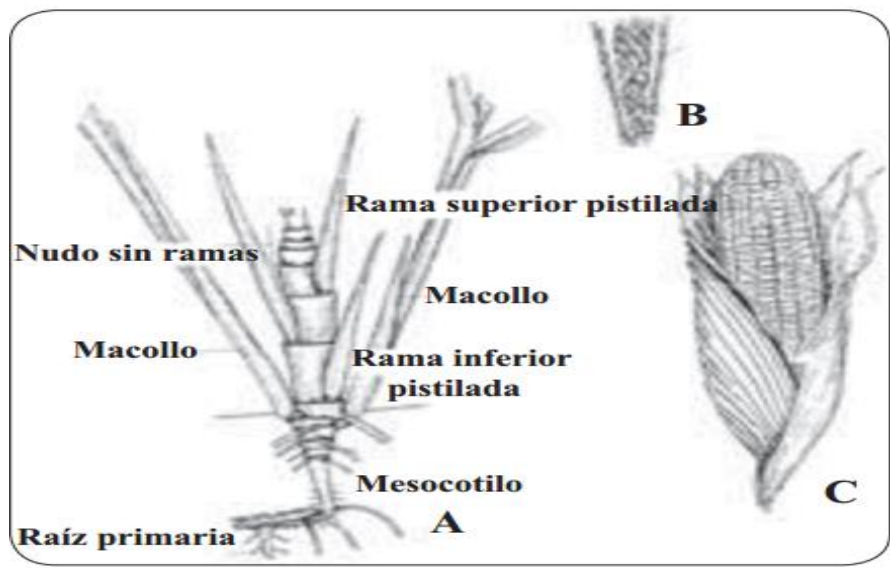

Figura[m4] 1: Maíz: características botánicas $A$. Planta, parte basal. B. Inflorescencia masculina. $C$. Mazorca.

Fuente: Manrique, 1997 citado por [20, 23]

\subsection{3. Época y densidad de siembra}

La semilla debe ser de buena calidad, con germinación no menor del $95 \%$, el terreno debe estar bien mullido, con suficiente humedad, en algunos casos necesita riego antes de la siembra.
La siembra se puede hacer a surco corrido, pero más frecuente es al golpe: se colocan 3 a 5 semillas por golpe a una profundidad de 5 a $8 \mathrm{~cm}$. La elección de una correcta distancia de plantas es determinante para lograr altos rendimientos en maíz; para ello, resulta conveniente analizar variables de manejo agronómico tales como: calidad ambiental, fecha de siembra y tipo de variedad utilizado [26]. La densidad de siembra depende de la fertilidad del suelo y del objetivo (maíz morado 25 a $30 \mathrm{~kg} / \mathrm{ha}$ ).

El maíz se cultiva generalmente con riego; por ello se establecen los campos en diferentes épocas según la altitud: en la zona agroecológica Quechua baja, entre 1800 y 2500 msnm se puede sembrar todo el ano si se dispone de riego; si este es insuficiente, la siembra se efectúa entre agosto y octubre. La época de siembra dependerá de las condiciones climáticas.

Hay múltiples factores que afectan la relación rendimiento-densidad. El genotipo, riego, cantidad de lluvia (abastecimiento de agua), nutrientes vegetales disponibles (suelo, además de fertilizantes), y la fecha de siembra se encuentran entre los principales factores que influyen en la relación rendimiento-densidad [1, 27-32].

Investigaciones previas sugieren que la densidad recomendada es de 60 mil plantas/ha el rendimiento aumenta en un $14,3 \%$ de producción de maíz para cultivo en secano y con buen drenaje del suministro de agua [33], y el tipo de suelo [34]. A diferencia de la mayoría de los cereales, el maíz no tiene la misma capacidad de ajustar el rendimiento para una posible insuficiencia ocasional o disponibilidad de los recursos en el suelo, el maíz puede producir un mazorca por planta, es decir, llegar a producir 1,890 a $8,980 \mathrm{~kg} / \mathrm{ha}$ y 4.56 a 10.2 plantas $/ \mathrm{m}^{2}$, respectivamente [33, 35]. Por lo tanto, el rendimiento de maíz, en particular maíz morado, depende de la densidad de plantas.

\subsubsection{Fertilización en maíz}

Materia orgánica (más de 2.5\%). El maíz es uno de los pocos cultivos andinos que siempre se fertiliza, generalmente con abundante estiércol (guano) y se añaden fertilizantes como urea y fósforo a niveles, 
de $80-80-0^{1}$ y en algunos casos en cantidades mayores, según tipo de suelos [20].

Tabla 2 - Requerimientos de fertilización en variedades de maíz morado PVM-581, PVM-582

\begin{tabular}{lclc}
\hline \multirow{2}{*}{ Época } & \multicolumn{3}{c}{ Fertilización } \\
\cline { 2 - 4 } & $\begin{array}{l}\text { Urea } \\
(\mathrm{kg})\end{array}$ & $\begin{array}{l}\text { Super } \\
\text { Fosfato } \\
\text { Triple } \\
(\mathrm{kg})\end{array}$ & $\begin{array}{c}\text { Cloruro de } \\
\text { Potasio }(\mathrm{kg})\end{array}$ \\
\hline $\begin{array}{l}\text { Siembra } \\
\text { Aporque }\end{array}$ & $\begin{array}{l}195.65 \\
\text { Inicio } \\
\text { floración }\end{array}$ & 217.39 & 100 \\
$\begin{array}{l}\text { Total } \\
\text { Total }\end{array}$ & 586.96 & 217.39 & 100 \\
\hline
\end{tabular}

Fuente: Lazo R., 1999; Zevallos M. [6]

\subsubsection{Deshierbo y aporques}

El maíz se deshierba al momento de aporcar, se pueden hacer tantas veces sea necesario, en el caso de que el cultivo sea solo de maíz [20, 36]. La primera se efectúa a los 45 días después de siembra, hasta el momento de la cosecha se realizan al menos dos deshierbas complementarias. Se hacen dos aporques durante los cuales también se eliminan las malezas y se evita la competencia por nutrientes, $\mathrm{H}_{2} \mathrm{O}$ y espacio.

\subsubsection{Control de plagas y enfermedades [20]}

\subsubsection{Plagas:}

La mosca de la mazorca: Ataca la punta de los granos: Euxesta sp. (Gusano de carne). Su acción favorece la presencia de hongos como Fusarium y Diplodia, provocan grandes pudriciones de la mazorca, el control se realiza con aceite, la mosca cebra Euxesta sp, ya no pone sus huevos en los pelos de la mazorca.

Para el control del gorgojo (Sitophilus orizae, Pagiocerus frontalis) y polilla (Calandragranaría, Sitotroga) se debe realizar en el almacén. Se controlan mediante la aplicación de Phostoxin utilizando 3 pastillas por tonelada de mazorcas y Gastión, 2 pastillas por tonelada de mazorcas.

\subsubsection{Enfermedades:}

\footnotetext{
${ }^{1}$ Es recomendable hacer una aplicación de 10 TM de materia orgánica a la preparación de terreno. Niveles de fertilización de: 270-100-60 [43].
}

Pudrición de la raíz Pythium sp.y bacterias Tizon Helminthosporium sp. Roya de la hoja Puccinia sorghi, enfermedad causada por fitoplasmas yespiroplasmas, el uso de variedades tolerantes.

Carbón del maíz (Ustilago maydis)

1) Sacar las mazorcas con agallas en estado verde para enterrarlas junto con guano de corral para compost. 2) rotación de cultivos es una práctica que permite disminuir la incidencia de esta enfermedad.

Achaparramiento (Puka poncho): presencia de franjas blanquecinas en la base de las hojas próximas a la inserción con el tallo, se prolongan hacia el ápice. Entrenudos cortos, enanismo de plantas sin granos, las mazorcas presentan granos flojos, pequeños, descoloridos o manchados. En caso de fitoplasma los síntomas foliares típicos son enrojecimiento de los márgenes de las hojas pudiendo más adelante cubrir toda su extensión. Sembrar variedades tolerantes.

\section{Enfermedades en la planta:}

Gusano de la mazorca (pulush): Heliothis zea. Aplicación de 3 gotas de aceite (6 litros/ha) de consumo humano en la parte apical de la mazorca cuando se observan posturas o larvas del primer estadío en el $10 \%$ de plantas. La aplicación es 2 litros en el primer tercio de floración, 2 litros en el segundo tercio de floración y 2 litros en el último tercio de floración (INIA, 2016). Aplicación de cebos envenenados al pie de la planta preparados en base afrechillo, melaza Carbaryl.

Gusano de tierra o cortador: Copitarsia turbata. (Agrotis ipsilon). La práctica cultural de riego de machaco, permite el ahogamientode las larvas antes de la preparación del suelo; larotación de cultivos es otra práctica que permite disminuirla población de estos insectos (Solid Perú, 2007).

Cogollero comedor de las hojas (utuscuro): Spodoptera fragiperda. Se debe hacer buena preparación previa a la siembra. Aplicación de Dípterex 2,5 g a razón de $10 \mathrm{~kg} / \mathrm{ha}$, aplicación de cipermetrinas a razón de 150 a 200 ml por cilindrode 200 litros de agua, cuando las larvas están raspando las hojas. Aplicación de cenizas también es efectivo (método ancestral).

\subsubsection{Cosecha}


El momento de cosecha se determina cuando las hojas de la planta muestran un amarillamiento y comienza el secado de las hojas inferiores. Tener en cuenta la época de lluvia que puede causar la pudrición de las mazorcas. La cosecha de maíz morado se realiza cuando la planta alcanza la madurez fisiológica es decir humedad del 30 al 35\% (Solid Perú, 2007). Siguiendo la tradición de nuestro ancestros se realizó la "tumba" que consiste en dejar caer las plantas secas con mazorcas incluidas por 4 días, luego se realiza la separación de la mazorca de la planta manualmente.

De manera general, según investigaciones, el rendimiento del maíz depende a la tecnología utilizada. Con tecnología media es de $4675 \mathrm{~kg} / \mathrm{ha}$, por encima de ello la región de Cajamarca presenta el mayor rendimiento a nivel nacional, con 8,39 $\mathrm{kg} / \mathrm{ha}$, estándar superior al promedio nacional. Apurímac y Huánuco también tienen rendimientos altos en este cultivo, llegando en el 2006 a 8,1 y 7,1 $\mathrm{Tm} /$ ha. Vale mencionar que, se ha encontrado rendimientos de $3.9 \mathrm{Tm} / \mathrm{ha}$ en Ayacucho y es inferior al promedio nacional [45]. En el ranking nacional, los menores resultados de rendimiento se obtienen en las regiones de Ayacucho Moquegua, Lambayeque y Lima (Solid - Perú, 2012). Con densidad de siembra de 8200/ha, abonamiento a razón de 270 100 - 60 y de $586.96 \mathrm{~kg}$ de urea, $217.39 \mathrm{~kg}$ de super fosfato triple y $100 \mathrm{~kg}$ de cloruro de potasio se han encontrado rendimientos de maíz morado entre $5000-3000 \mathrm{~kg} / \mathrm{ha}[43,44]$.

\subsection{Estados vegetativos del maíz}

Dentro del desarrollo de los estados fenológico del maíz ocurren eventos importantes en ciertos estados, que se mencionan a continuación:

V3: El punto de crecimiento está bajo tierra, las bajas temperaturas pueden aumentar el tiempo entre la aparición de las hojas y el daño por helada en este estado tiene muy poco efecto en el crecimiento y rendimiento final.

V6: Se completa la fertilización, puesto que el sistema de raíces nodales está bien distribuido en el suelo. También es posible observar síntomas de deficiencias de macro o micro nutrientes.

V9: Varias mazorcas rudimentarias ya se encuentran formadas, la panoja se desarrolla rápidamente en el interior de la planta. Además comienza una rápida acumulación de biomasa, absorción de nutrientes y agua que continuará hasta casi el término del estado reproductivo.

V12: Se determina el tamaño potencial de mazorca y número potencial de óvulos por mazorca. Dado que se está formando el tamaño de mazorca y número de óvulos, riego y nutrición son críticos.

V15: Es el estado más crucial para la determinación del rendimiento. Las hojas aparecen cada uno o dos días y las sedas están comenzando a crecer en las mazorcas superiores.

R1: El número de óvulos fertilizados se determina en este estado. Los óvulos no fertilizados no producen grano y mueren. El estrés ambiental en este momento afecta la polinización y cuaje, especialmente el estrés hídrico que deseca las sedas y los granos de polen. Además, a partir del inicio de este estado hasta $\mathrm{R} 5$ se produce un rápido llenado del grano por lo que se presenta también ataque de gusano por lo que es necesario realizar controles.

R5: Los granos empiezan a secarse desde la parte superior donde se forma una capa blanca de almidón. El estrés y las heladas pueden reducir el peso de los granos. Llegando a $\mathrm{R} 6$ donde el grano alcanza su peso máximo y es cosechado.

Cuadro 2 - Caracterización de los estados vegetativos y reproductivos del maíz morado

\begin{tabular}{ll}
\hline Estados vegetativos & \multicolumn{1}{c}{ Estados reproductivos } \\
\hline Ve : emergencia & R1 : sedas \\
V1 :primera hoja & R2 : ampolla \\
V2 :segunda hoja & R3 : Grano lechoso \\
V3 : tercera hoja & R4 : Grano pastoso \\
V(n):enésima hoja & R5 : Dentado \\
VT : Panoja & R6 : Madurez Fisiológica \\
\hline
\end{tabular}

\subsection{Variedades de maíz morado}

Perú alberga cerca de 36 ecotipos de variedades de maíz (FAO, 2016) de 55 especies y en Bolivia 31; de estas destaca el maíz amarillo duro cultivado en la costa, el maíz amiláceo de la sierra para choclo, cancha y maíz morado [20]. Manrique (1997), las variedades de maíz morado se caracteriza de la siguiente manera: Morado Canteño, Morado Mejorado, Morado Caraz, Arequipeño, Cuzco Morado y Negro Junín. Sin embargo, la variedad más comercial es el maíz Morado Canteño porque se desarrolla bien desde 1800 a 2500 msnm, con floración entre 110a 125 días, tolerante a plagas, enfermedades y se adapta a diferentes zonas, y podemos encontrar en la sierra a los 3000 msnm [37]. El maíz de color morado, es una variedad genética de Perú; una mazorca (coronta y grano) constituye el $85 \%$ y $15 \%$ por coronta (tusa), esta planta contiene el pigmento denominado antocianina, la mayor cantidad se encuentra en la coronta y en menor proporción en el pericarpio del grano, siendo uno de los principales alimentos en la 
dieta peruana, utilizado frecuentemente en preparación de bebidas como la chicha morada y postres como la mazamorra morada [38]. A nivel nacional Quispe, F. et al (2011), estudiaron las características morfológicas y químicas de los cultivares TC, PM 581 y TJ de maíz morado, cultivados en el distrito de La Joya-Arequipa. Los resultados indican diferencias altamente significativas $(p<0.01)$ para altura de planta a los 8 , $15,30,60,90,120$ y 150 días después de siembra; diferencias significativas $(p<0.05)$ para peso de grano y los caracteres morfológicos: diámetro de mazorca, diámetro de coronta y peso de coronta. El análisis de antocianinas y fenoles totales de las corontas muestra diferencias significativas en $p<0,05$, sobresaliendo $P M-581$. El análisis proximal de los granos se encontró dentro de los valores reportados en la literatura [10].

\subsection{Variedades en estudio de maíz morado}

\subsubsection{Variedad INIA - 601 (INIA NEGRO):}

Se originó en 1990 en la Subestación Experimental Cajabamba, región Cajamarca Perú [45]. INIA NEGRO se formó con 256 progenies, 108 de la variedad Morado de Caráz y 148 progenies de la variedad local Negro de Parubamba (INIA Baños del Inca, 2014). Tiene № de registro en SENASA 01/2000-AG-SENASA-DGSV, entidad que registró INIA, con fecha 29-Mar-00, es de ámbito Sierra Norte del Perú [8]; SENASA, 2008). Esta especie incide fundamentalmente en el color morado intenso de coronta y grano, precocidad, rendimiento, buen tipo de planta y sanidad de mazorcas. Tiene producción promedio de $0.20 \mathrm{TM} /$ ha/ciclo.

\section{Cultivo y manejo de la línea INIA - 601}

Es una variedad de polinización abierta adaptada a la sierra norte del Perú (Regiones de Cajamarca, La Libertad y Piura). Soporta alturas de 2600 y 2900 msnm. La siembra realizado en los meses de octubre y noviembre, obtiene 50000 plantas/ha, se recomienda emplear $35 \mathrm{~kg}$ de semilla de buena calidad (germinación y emergencia). Distanciar los surcos a 0,80 $\mathrm{m}$ y sembrar 3 semillas/golpe cada $0,50 \mathrm{~m}$ y al desahíje dejar 2 plantas/golpe. Antes de la siembra tratar $35 \mathrm{~kg}$ de semilla con $150 \mathrm{~g}$ de Orthene PS. Se debe eliminar oportunamente malezas haciendo un deshierbo oportuno. Si es posible utilizar herbicida a base de Atrazina en la dosis comercial recomendada, aplicando en terreno con humedad antes de la emergencia del maíz. Se mantiene limpio el cultivo hasta la floración. Fertilizar teniendo en cuenta el análisis de suelo. Se aplica la fórmula 90-45-00 kg/ha de $\mathrm{N}, \mathrm{P}_{2}, \mathrm{O}_{2}, \mathrm{k}_{5}, \mathrm{o}$ fraccionando la aplicación del nitrógeno en dos partes: un tercio en el primer deshierbo y dos tercios antes de la floración. Cuando el clima es seco, se presenta ataque de cogollero (Spodoptera frugiperda), para evitar los daños debe aplicarse insecticida granulado, Dipterex en la dosis comercial recomendada (INIA, 2016). Para evitar el ataque de los gusanos mazorqueros Heliothis zea y Euxesta spp, al momento de la floración, aplicar 3 gotas de aceite comestible a los pistilos con ayuda de un gotero o de un mechón de lana, en 3 oportunidades (con una semana de intervalo).

Tabla 3: Principales características agronómicas de la variedad INIA - 601

\begin{tabular}{|c|c|}
\hline Descripción & Datos \\
\hline Altura de planta & $2.16 \mathrm{~m}$ \\
\hline Altura de mazorca & $1.24 \mathrm{~m}$ \\
\hline $\begin{array}{l}\text { Días a la floración } \\
\text { femenina }\end{array}$ & 98 \\
\hline Días a la madurez & 170 \\
\hline $\begin{array}{l}\text { Unidades de calor a la } \\
\text { floración }\end{array}$ & $875.7 \pm 8,1^{\circ} \mathrm{Cd}$ \\
\hline Hojas normales & Lanceoladas \\
\hline $\begin{array}{l}\text { Número de hojas por } \\
\text { planta }\end{array}$ & 12 \\
\hline $\begin{array}{l}\text { Número de mazorcas } \\
\text { por planta }\end{array}$ & 1 a 2 \\
\hline Descripción & Datos \\
\hline Forma de la mazorca & Ligeramente cónica \\
\hline Color de la mazorca & Morado intenso \\
\hline Color de tusa & Morado \\
\hline Longitud de mazorca & $17.5 \mathrm{~cm}$ \\
\hline Diámetro de mazorca & $4.6 \mathrm{~cm}$ \\
\hline Número de hileras & 10 a 12 \\
\hline $\begin{array}{l}\text { Número de granos por } \\
\text { hilera }\end{array}$ & 26 \\
\hline Consistencia del grano & Harinosa \\
\hline Longitud del grano & $13 \mathrm{~mm}$ \\
\hline Ancho del grano & $11 \mathrm{~mm}$ \\
\hline Espesor del grano & $5 \mathrm{~mm}$ \\
\hline $\begin{array}{l}\text { Porcentaje } \\
\text { desgrane }\end{array}$ & $78 \%$ \\
\hline Peso de 1000 semillas & $456.2 \mathrm{~g}$ \\
\hline $\begin{array}{ll}\text { Potencial } & \text { de } \\
\text { rendimiento } & \end{array}$ & 6.0 TM/ha \\
\hline $\begin{array}{l}\text { Rendimiento en campo } \\
\text { de Agricultor }\end{array}$ & 3.0 TM/ha \\
\hline
\end{tabular}


fecundación, período de fecundación a maduración fisiológica. Se recomienda riego por gravedad ${ }^{2}$; esto

La dosis es de $3 \mathrm{~L}$ de aceite/ha/aplicación. La cosecha de mazorcas realiza en plena madurez fisiológica con un máximo de $15 \%$ humedad de grano (INIA, 2014). Del INIA - 601, los estudios llevados a cabo en el INIAP-H-601 Ecuador se han obtenido rendimientos de $7381 \mathrm{~kg} / \mathrm{ha}$.

La línea INIA - 601, para siembra en sierra media, su floración femenina ocurre de 90 a 105 días después de la siembra [42].

\subsubsection{Variedad UNC - 47:}

Es una nueva variedad de maíz morado, obtenida por la Universidad Nacional de Cajamarca (UNC) tras 11 años de estudio en el programa de Investigación de Maíz de la Facultad de Ciencias Agrarias, consigue un buen rendimiento en cuanto a coloración y sus cualidades medicinales principalmente en la coronta (Tirado Zoto, C. et al., 2000) citado por [45]. Las mejores épocas de siembra son en octubre y noviembre para obtener mejores rendimientos. Esta variedad destaca porque produce mazorcas de excelente calidad en pigmentación (morado intenso) 190 días después de la siembra, debido a la concentración de antocianinas que se encuentra en la coronta. La importancia del cultivo es que solo se produce en Perú, Bolivia y Ecuador.

\subsection{3. $P V M-581:$}

Es una variedad derivada del maíz Caraz. Se siembra en la costa central y en sierra media [43], su floración femenina ocurre de 55 a 60 días después de la siembra [42].

Tolera suelos profundos de textura franca a francoarcilloso, con buena capacidad para retener humedad, no deben presentar problemas de drenaje; excesos de humedad son adversos a la acumulación de pigmentos en la mazorca, $\mathrm{pH}$ : 5-8, conductividad eléctrica entre: 1-4 dS/m [43]. Soporta alturas de 1,200-4,000 msnm. Para siembra en golpes (3 semillas/golpe), $0.70 \mathrm{~m}$ entre surcos y $0.55 \mathrm{~m}$ entre golpes. Para siembra en hilera: una planta cada $0.15 \mathrm{~m}$ y $0.80 \mathrm{~m}$ entre surcos. La densidad aproximada debe ser de 8200 plantas/ha. Las etapas del cultivo se desenvuelven tal como para las variedades comenzando por: Emergencia y establecimiento, período de siembra a germinación, período de germinación a aporque, período de aporque a floración, período de

\footnotetext{
2 Hacer el riego cada 10 a 12 días a razón de 8,000 a
} $10,000 \mathrm{~m}^{3} /$ ha (Lazo, R. 1999). varía según el clima y tipo de suelo. Priorizar riegos durante la floración y panojamiento [43]. Tiene № de registro en SENASA 008, entidad que registró DRA Cusco [47], con fecha 27-Jun-83, es de ámbito Costa y sierra, baja y media del Perú [8]; SENASA, 2008).

\section{Método y procedimiento}

Previo se realizó el análisis del suelo, por lo que se tomaron muestras entre $0-30 \mathrm{~cm}$ de profundidad, el cual se envió al Laboratorio de Investigación de Suelos y Aguas del INDES-CES - UNTRM. Las labores culturales, comenzando por la preparación del terreno, siembra, manejo del cultivo y cosecha. La investigación se llevó a cabo en el anexo Quipachacha, distrito de Levanto, en las coordenadas N 6 $37^{\prime} 37^{\prime \prime} \mathrm{S}, 77^{\circ} 49^{\prime} 1$, a una altitud de $2820 \mathrm{msnm}$ en la Estación Experimental UNTRM, INDES- CES. Asimismo el estudio detalla el efecto de tres distanciamientos entre plantas y surcos respectivamente: $40 \mathrm{~cm} \times 60 \mathrm{~cm}, 30 \mathrm{~cm} \times 50 \mathrm{~cm}$ y $20 \mathrm{~cm} \times 60 \mathrm{~cm}$; con el comportamiento agronómico además se contó con un testigo $40 \mathrm{~cm} \times 60 \mathrm{~cm}$ (datos de distanciamiento del agricultor) sin abonamiento.

\subsection{Población y muestra}

La población total estuvo conformada por tres variedades de maíz morado Zea mays L. (INIA 601), (UNC - 47) y (PVM - 581), cultivadas bajo las condiciones del anexo de Quipachacha entre los meses de diciembre del 2015 a Julio del 2016, distrito de Levanto, provincia de Chachapoyas, región Amazonas, Perú. La muestra fueron los 9 tratamientos los cuales fueron evaluados el efecto de tres densidades $\left(2.5,1.5\right.$ y $\left.1.2 \mathrm{~m}^{2}\right)$ de siembra en el comportamiento agronómico de maíz por triplicado. El diseño fue en Bloque Completo al Azar - DBCA y para la contrastación de la hipótesis se usó un la prueba Tukey con el programa SPSS Statistics (Versión 24.0). Se consideró un testigo absoluto (densidad de $1.20 \mathrm{~m}^{2}$ ) densidad común del agricultor.

La muestra se determinó de acuerdo a la densidad de siembra, usando fórmula estadística de proporciones para población finita. 


\section{2. Área del terreno, y distribución de las parcelas experimentales}

El área de la parcela experimental fue de $844,8 \mathrm{~m}^{2}$, se consideró 3 bloques para el experimento, los cuales estaban conformados por 9 parcelas demostrativas con una distancia de separación entre bloques de $1.5 \mathrm{~m}$ y $1 \mathrm{~m}$ entre parcelas 0 unidad experimental que tuvo un área de $13.2 \mathrm{~m} \times 64 \mathrm{~m}$ tal como se muestra en la tabla siguiente:

\section{Tabla 4: Características de las parcelas experimentales}

\begin{tabular}{|c|c|}
\hline Experimento factorial & DBCA \\
\hline № de tratamientos & 9 \\
\hline № de bloques ${ }^{3}$ & 3 \\
\hline $\mathrm{N}^{\circ}$ total de observaciones & 27 \\
\hline $\begin{array}{l}\text { Distanciamiento entre } \\
\text { surcos }\end{array}$ & $60 \mathrm{~cm}, 50 \mathrm{~cm}$ y $60 \mathrm{~cm}$ \\
\hline $\begin{array}{l}\text { Distanciamiento entre } \\
\text { plantas }\end{array}$ & $40 \mathrm{~cm}, 30 \mathrm{~cm}$ y $20 \mathrm{~cm}$ \\
\hline $\begin{array}{l}\text { № de surcos/unidad } \\
\text { experimental }\end{array}$ & 5,7 y 8 \\
\hline № de plantas/surco & $\mathrm{d} 1=13, \mathrm{~d} 2=17$ y d3=26 \\
\hline № de plantas a evaluar & $d 1=10, d 2=10$ y d3 $=10$ \\
\hline Separación entre bloques & $1,5 \mathrm{~m}$ \\
\hline $\begin{array}{l}\text { Separación entre parcelas } \\
\text { (tratamientos) }\end{array}$ & $1 \mathrm{~m}$ \\
\hline Longitud de los tratamientos & $3.4 \mathrm{~m}$ \\
\hline Área total del experimento & $844.8 \mathrm{~m} 2$ \\
\hline
\end{tabular}

\subsection{Diseño estadístico del campo experimental}

En esta investigación se trabajó con un diseño Factorial en Bloque Completamente al Azar (DBCA) con 9 tratamientos, se utilizó el paquete estadístico SPSS Statistics (versión 24.0).

Tabla 5: Tabla de unidades experimentales ${ }^{4}$

\begin{tabular}{lcccc}
\hline $\begin{array}{c}\text { cantidad } \\
\text { parcela }\end{array}$ & $\begin{array}{c}\text { variedad de } \\
\text { maíz morado }\end{array}$ & $\begin{array}{c}\text { densidad } \\
\mathrm{cm}\end{array}$ & $\begin{array}{c}\text { densidad } \\
\text { de } \\
\text { siembra } \\
\left(\mathrm{m}^{2}\right)\end{array}$ & Trat. \\
\hline 1 & INIA 601 & $60 \times 40$ & 2.4 & T1 \\
2 & UNC -47 & $60 \times 40$ & 2.4 & T2 \\
3 & PVM -581 & $60 \times 40$ & 2.4 & T3 \\
4 & INIA 601 & $50 \times 30$ & 1.5 & T4 \\
5 & UNC - 47 & $50 \times 30$ & 1.5 & T5 \\
6 & PVM -581 & $50 \times 30$ & 1.5 & T6 \\
7 & INIA 601 & $60 \times 20$ & 1.2 & T7 \\
8 & UNC - 47 & $60 \times 20$ & 1.2 & T8 \\
9 & PVM -581 & $60 \times 20$ & 1.2 & T9 \\
\hline
\end{tabular}

3 Pendiente del terreno, considerado también como repeticiones de los tratamientos (3 repeticiones)

\subsection{Instalación y Conducción de las Parcelas Experimentales}

\section{Muestreo de suelo:}

Es necesario conocer las características fisicoquímicas del suelo (textura, $\mathrm{pH}$, conductividad eléctrica y macro nutrientes NPK), por lo que se realizó la recolección de muestra del suelo un mes antes de la instalación, usando el método de Zig Zag [39], tratando de cubrir todo el área de la investigación, de forma mecánica con ayuda de una palana, a través de una calicata. Luego, se realizó la aplicación de fertilizante de acuerdo al requerimiento de cultivo.

\subsection{Preparación del terreno y aplicación de cal agrícola}

Se hizo el reconocimiento del terreno de $844.8 \mathrm{~m}^{2}$, se realizó la limpieza de malezas para favorecer la germinación y enraizamiento; y se procedió a realizar las siguientes labores: arado del terreno con motocultor. A los 3 días se agregó mezcla de cal con guano de isla de acuerdo a los requerimientos del maíz según el estudio de suelo; se dejó 15 días para la descomposición de los desechos vegetales y asimilación con el encalado (equilibrio en el pH al suelo). Seguidamente se hizo la cruza con la finalidad de desmenuzar los terrones grandes del suelo, delimitación y trazado del terreno surcado $60 \times 40 \mathrm{~cm}$, y demás densidades de siembra.

Tabla 6: Fertilizantes químicos utilizados en el experimento

\begin{tabular}{lccc}
\hline Fertilizante & \multicolumn{3}{c}{ \% fertilizante } \\
& $\mathrm{N}$ & $\mathrm{P}$ & $\mathrm{K}$ \\
\hline Urea & 46 & - & - \\
Fosfato Diamónico & 18 & 46 & - \\
{$\left[\left(\mathrm{NH}_{4}\right)_{2} \mathrm{HPO}_{4}\right]$} & & & \\
Cloruro de Potasio & - & - & 60 \\
{$[\mathrm{KCl}]$} & & \\
Dosis de fertilización $\left(\mathrm{N}-\mathrm{P}_{2} \mathrm{O}_{5}-\mathrm{K}_{2} \mathrm{O}\right)$ & \\
q1 & $140-120-100$ \\
q2 & $160-140-120$ \\
q3 & $180-160-140$ \\
\hline
\end{tabular}

${ }^{4}$ Las repeticiones, vienen hacer los bloques que son la pendiente del terreno 


\subsubsection{Abonamiento: aplicación de fertilizante}

Se aplicó materia orgánica (guano de isla) $120-80$ - $60 \mathrm{~kg} / \mathrm{ha}$ de $\mathrm{N}, \mathrm{P}_{2} \mathrm{O}_{5}$ y $\mathrm{K}_{2} \mathrm{O}$ respectivamente de acuerdo a la correlación con el análisis de suelo. El primer abonamiento se realizó al momento de siembra se aplicó la mitad de nitrógeno y todo el fósforo, potasio. El segundo abonamiento nitrogenado se realizó al momento del aporque en el desarrollo vegetativo V6 (plantas entre 8 a 10 hojas extendidas), en los valles interandinos esto ocurre a los 30 a 40 días después de la siembra [42].

\subsubsection{Siembra de maíz morado}

Se usó semilla ${ }^{5}$ por lo que se presume que tendrá un alto porcentaje de germinación, con buen vigor y libre de enfermedades. Se sembraron 3 semillas por golpe, el desahíje se realizó entre los 12 y 15 días, quedando solo una planta vigorosa. Se usó entre 1 $1 / 2 \mathrm{~kg}$ de semilla/ha por variedad. La profundidad de siembra fue de $6 \mathrm{~cm}$ por estaca, dado que la textura del suelo es franco arenoso llegando hasta $10 \mathrm{~cm}$ en suelo arenosos [43, 44$]$.

\subsubsection{Labores culturales en el maíz morado}

\section{Primer y segundo deshierbo (cutipa) y aporque:}

El deshierbo se realizó con el fin de evitar la competencia de nutrientes, luz, espacio entre el crecimiento de la planta. Las malezas son hospedadoras de plagas y enfermedades, en los primeros 14 días se realizó dos limpiezas culturales, junto se realizó la primera fertilización. La actividad se realizó manualmente usando lampas. El segundo deshierbo se realizó a los 37 días, junto con el aporque, esta última actividad consiste en juntar el suelo en la base de la planta (la altura de la planta alcanzó entre $50-60 \mathrm{~cm}$ ) esta labor ayuda a precisar las raíces, permite eliminar malezas que han conseguido desarrollarse alrededor de la planta del maíz morado. En esta etapa también se realizó el segundo abonamiento nitrogenado.

\subsubsection{Riego}

La presencia de sequías es común en los valles de Quipachacha en Levanto por lo que obligó a completar el periodo vegetativo con riego en el momento crítico cada 12 días: durante el establecimiento del cultivo (se consigue de

\footnotetext{
5 Semilla certificada. Categoría autorizada: № 077124.
} Cultivar: INIA 601. Lote №: 01 LAM3-07014. Productor: antemano con el riego de machaco antes de la preparación del terreno), antes del aporque, en la etapa de floración con mayor periodización y llenado de grano (panojamiento). El volumen de agua usado fue de 8 a $10 \mathrm{mil} \mathrm{m}^{3} / \mathrm{ha}$ (INIA, 2016).

\subsection{5. Época de seca, cosecha y almacenamiento}

La mazorca se dejó secar hasta llegar al $14 \%$ de contenido de humedad con buena ventilación $(60 \%$ HR); para ello, primero se realizó la selección, el procedimiento fue rápido, con energía solar, sin que llegue directamente a las mazorcas (se colocó en el techo bajo calamina) para no perder las antocianinas. Lejos de infectarse por hongos, gorgojos o ser consumidos por roedores. Se usó 3 pastillas de Phostoxin por tonelada de mazorca almacenada [42] para evitar la proliferación de la polilla de almacén ya que a partir de esta época si no se controla comienza la infestación.

\subsection{Variables evaluadas:}

Fue necesario realizar evaluaciones cada 20 días posterior a la siembra, a fin de observar y medir los diferentes parámetros del cultivo [7].

\subsubsection{Porcentaje de germinación:}

Se realizó para tener la seguridad que la mayor parte de semillas sembradas en las parcelas, germinaron; se observó a los 8 días.

\subsubsection{Número de plantas por metro lineal (NM- $M L):$}

Se evaluaron solo 10 plantas por parcela, de acuerdo a las densidades especificadas, al $100 \%$ de emergencia de plántulas, esto con el fin de determinar el número de plantas/ha. A los 15 días después de haber instalado en campo definitivo se evaluó el estado vegetativo (VE - emergencia).

\subsubsection{Altura de planta (AP):}

Se tomaron tres surcos centrales de cada parcela obteniendo el número de plantas de acuerdo a las densidades evaluadas. Identificadas desde la base del tallo hasta la hoja bandera y floración. La medición se realizó a las mismas plantas cuando ellas poseían 6 hojas, aproximadamente (entre 20 25 días). La formación de la panoja a los 25 a 35

INIA Baños del Inca. Fecha: Noviembre 2015. Lugar de Producción Cajamarca. 
días crecimiento, y el total de hojas entre 45 a 60

días.

Tabla 7-Dosis de fertilización de acuerdo a la densidad de siembra y estudio de suelo ${ }^{6}$

\begin{tabular}{|c|c|c|c|c|c|c|c|c|c|}
\hline 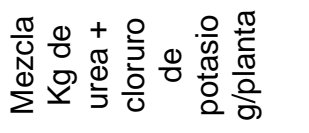 & 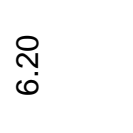 & $\stackrel{+}{\stackrel{N}{r}}$ & $\begin{array}{l}\stackrel{\infty}{ஸ ̣} \\
\text { ஸे }\end{array}$ & $\begin{array}{l}\stackrel{L}{6} \\
\stackrel{+}{+}\end{array}$ & 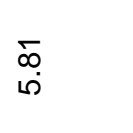 & $\begin{array}{l}\mathscr{\leftrightarrow} \\
\stackrel{\circ}{\circ}\end{array}$ & $\frac{\circ}{\dot{m}}$ & $\begin{array}{c}\hat{\infty} \\
\stackrel{m}{ }\end{array}$ & $\begin{array}{l}\dot{J} \\
\dot{\forall}\end{array}$ \\
\hline 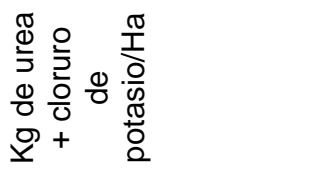 & 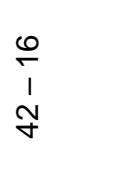 & 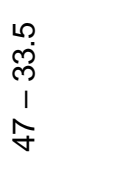 & $\begin{array}{l}\text { g } \\
1 \\
\text { ஸ̃ }\end{array}$ & 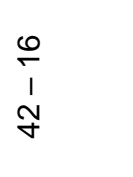 & 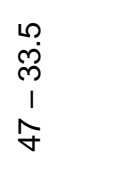 & $\begin{array}{l}\text { g } \\
\text { I } \\
\text { ̃ }\end{array}$ & 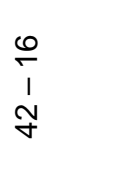 & 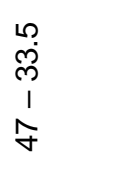 & $\begin{array}{l}\text { g } \\
1 \\
\text { ஸ̃ }\end{array}$ \\
\hline 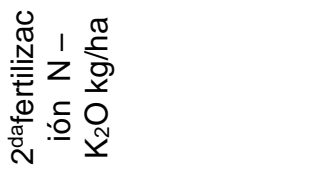 & $\begin{array}{l}\text { 우 } \\
\sigma \\
1 \\
\dot{0}\end{array}$ & 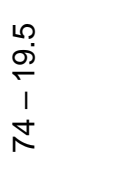 & $\begin{array}{l}0 \\
\stackrel{D}{\infty} \\
1 \\
1 \\
\infty\end{array}$ & $\begin{array}{l}\text { 우 } \\
\text { ô } \\
1 \\
8 \\
0\end{array}$ & 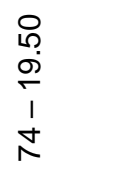 & $\begin{array}{l}0 \\
\stackrel{1}{0} \\
\text { N } \\
1 \\
\infty \\
\infty\end{array}$ & $\begin{array}{l}\text { 우 } \\
0 \\
1 \\
\vdots \\
0\end{array}$ & $\begin{array}{l}\frac{1}{2} \\
\frac{1}{1} \\
0 \\
0 \\
0\end{array}$ & $\begin{array}{l}\text { O } \\
\stackrel{\infty}{\infty} \\
\text { 1 } \\
1 \\
\infty\end{array}$ \\
\hline 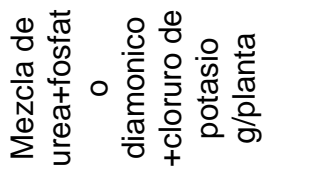 & $\stackrel{\stackrel{N}{N}}{\stackrel{N}{\sim}}$ & $\begin{array}{l}\stackrel{\leftrightarrow}{\circ} \\
\dot{+}\end{array}$ & $\stackrel{\text { \& }}{\stackrel{+}{\Sigma}}$ & $\frac{\infty}{\sigma}$ & $\stackrel{m}{\leftarrow}$ & $\frac{m}{m}$ & $\frac{1}{6}$ & 文 & $\begin{array}{l}\stackrel{N}{N} \\
\infty\end{array}$ \\
\hline 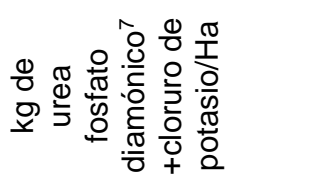 & \begin{tabular}{l}
1 \\
$\stackrel{\infty}{+}$ \\
$\stackrel{1}{\sim}$ \\
1 \\
\multirow{y}{*}{}
\end{tabular} & 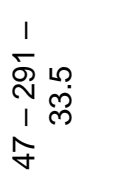 & 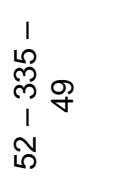 & $\begin{array}{l}1 \\
\infty \\
\stackrel{\infty}{+} \\
1 \\
\stackrel{y}{y}\end{array}$ & 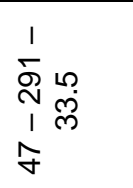 & 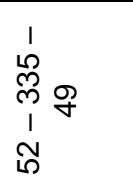 & \begin{tabular}{l}
1 \\
$\stackrel{\infty}{+}$ \\
$\stackrel{1}{*}$ \\
1 \\
\multirow{f}{*}{}
\end{tabular} & 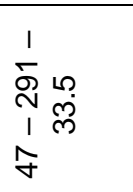 & 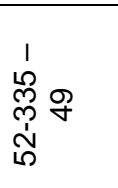 \\
\hline 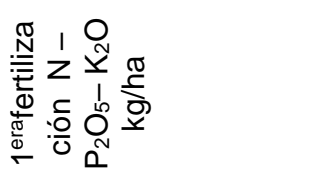 & 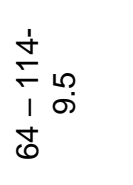 & 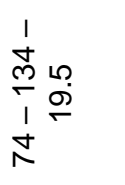 & 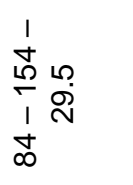 & \begin{tabular}{l}
1 \\
\multirow{2}{*}{} \\
$\begin{array}{l}1 \\
1\end{array}$ \\
0 \\
0
\end{tabular} & 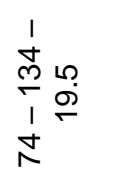 & 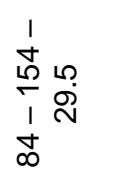 & 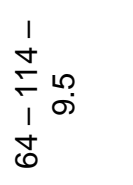 & 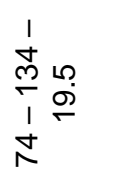 & 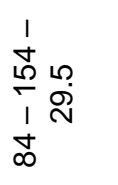 \\
\hline 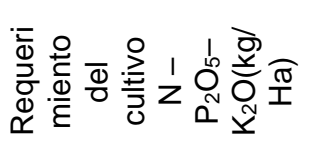 & 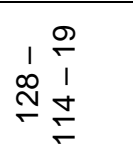 & 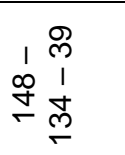 & 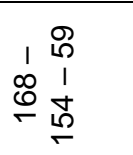 & 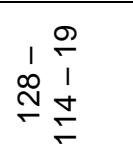 & 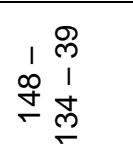 & 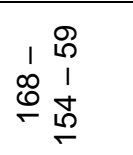 & 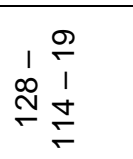 & 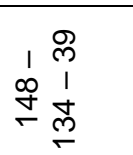 & 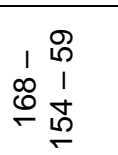 \\
\hline 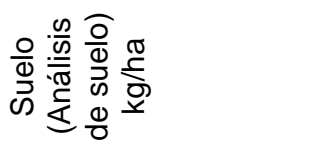 & 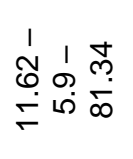 & 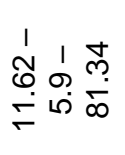 & 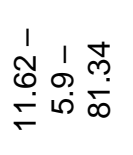 & 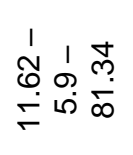 & 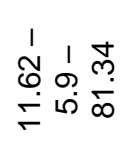 & 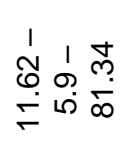 & 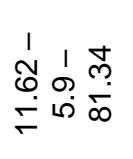 & 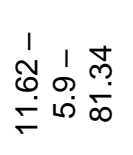 & 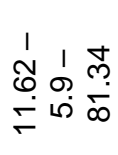 \\
\hline 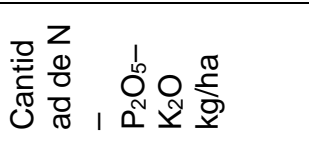 & 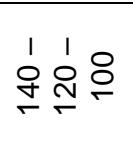 & 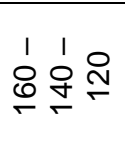 & \begin{tabular}{ll}
1 & 1 \\
0 & 0 \\
0 & 0 \\
\hdashline & +
\end{tabular} & 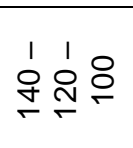 & 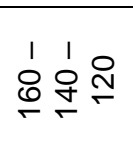 & \begin{tabular}{ll}
1 & 1 \\
$\infty$ & 0 \\
\hdashline & 0 \\
\hdashline
\end{tabular} & 맘용으 & 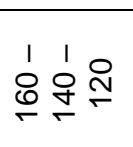 & \begin{tabular}{ll}
1 & 1 \\
$\infty$ & 0 \\
$\infty$ & 0 \\
\hdashline & 0 \\
-
\end{tabular} \\
\hline 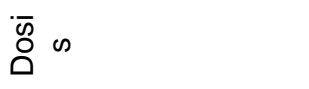 & $\bar{\sigma}$ & $\widetilde{O}$ & $\ddot{\sigma}$ & $\bar{\sigma}$ & $\widetilde{\sigma}$ & $\ddot{g}$ & $\bar{\sigma}$ & $\widetilde{\sigma}$ & $\ddot{O}$ \\
\hline 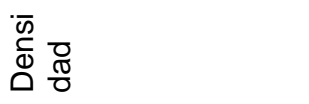 & ㄷ & ㄷ & ㄷ & 긍 & 임 & 긍 & ஜㅁ & றृ & M \\
\hline 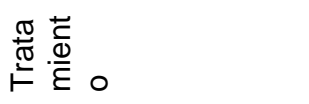 & $F$ & $\stackrel{\sim}{\vdash}$ & $\stackrel{\mathscr{P}}{\circ}$ & $\stackrel{\forall}{\vdash}$ & $\stackrel{\sim}{\vdash}$ & $\stackrel{\bullet}{\bullet}$ & $F$ & $\stackrel{\infty}{\vdash}$ & $\stackrel{\circ}{\vdash}$ \\
\hline
\end{tabular}

6 Está en función del estudio de suelo realizado a la parcela 6. $\quad$ Fredy, Q.J., Características morfológicas y químicas de 3 cultivares de maíz morado (Zea mays L.) en Arequipa-Perú. Revista de la Sociedad Química del Perú, 2016. 77(3).\& Lazo, R. 1999).
$7\left[\left(\mathrm{NH}_{4}\right)_{2} \mathrm{HPO}_{4}\right] 40$. Bayer, C. and J. Mielniczuk, Características químicas do solo afetadas por métodos de preparo e sistemas de cultura. Revista brasileira de Ciência do Solo, 1997. 21(1): p. 105-112. 


\subsubsection{Días de floración (DF):}

Contando desde el día que se realizó la siembra, hasta la aparición de la floración de las plantas (93 días).

\subsubsection{Altura de inserción de mazorca [7]:}

De las mismas plantas en la que se midió altura, se midió la altura de carga de la mazorca, también desde el nivel del suelo hasta el nudo de la inserción de la mazorca principal su representación se realiza con promedio en $\mathrm{cm}$ a los 80 días.

\subsubsection{Número de mazorca por planta (NMP):}

Con finalidad de ver el número de mazorcas por cada planta y capacidad de variedad producida (rendimiento) de mazorcas se realizó a los 85 días.

\subsubsection{Número de hileras por mazorca (NIM):}

Para la estimación del rendimiento a los 90 días etapa reproductiva $\mathrm{R} 4$.

\subsubsection{Diámetro de mazorca (DM):}

Se utilizó un calibrador con el cual se midió el diámetro en el tercio medio de cada mazorca y unidad cm, justo en etapa reproductiva R5 a los 100 días.

\subsubsection{Longitud de la mazorca (LM):}

Del total de mazorcas cosechadas en cada parcela se tomó al azar 10 mazorcas para luego individualmente medir su longitud en $\mathrm{cm}$, desde la base hasta el ápice, esto en etapa reproductiva dentado $\mathrm{R} 5$ a los 100 días.

\subsubsection{Peso de Semillas 100 (PS) g}

De 10 plantas seleccionadas se escogieron 10 granos de mazorca, se realizó en cada tratamiento (parcela); libres de hongos, insectos e impurezas, se pesó en una balanza de precisión (etapa R6). El peso de los $100 \mathrm{~g}$ servirá para evaluar el rendimiento kg/planta, fórmula CIMMYT Perrin, 1998 citado por [3].

$$
\text { Rdto }\left(\frac{K g}{h a}=\frac{10000}{\mathrm{DSxDP}}\right) x \text { Rdto } / \text { planta }
$$

Donde: $\mathrm{DS}=$ distanciamientos entre surcos

$\mathrm{DP}=$ distanciamientos entre plantas

Se evaluó el rendimiento total de maíz morado por parcela experimental ajustándolo en un $10 \%$ menos, para reflejar los rendimientos que se podrían esperar en campo.

\subsection{Rendimiento}

Rendimiento por hectárea $(\mathrm{kg} / \mathrm{ha})$, entre los 115 y 120 días (etapa reproductiva de madures fisiológica = R6) se registró el peso de granos obtenidos en cada parcela útil, para ello se utilizó una balanza y la fórmula siguiente:

Donde :

$$
P u=\frac{P a(100-h a)}{(100-h d)}
$$

Pu: peso uniformizado Pa: peso actual ha: humedad actual hd: humedad deseada

\section{Resultados}

En la tabla 8 se observa el resultado de análisis de varianza de las variedades PVM - 581, INIA - 601 y UNC - 47 de maíz morado y sus variables agronómicas evaluadas a tres densidades de siembra $(60 \mathrm{~cm} \times 40 \mathrm{~cm}) 2.4 \mathrm{~m}^{2},(50 \mathrm{~cm} \times 30 \mathrm{~cm}) 1.5 \mathrm{~m}^{2}$ y $(60 \mathrm{~cm} \times 20 \mathrm{~cm}) 1.20 \mathrm{~m}^{2}$ al $\mathrm{p}<0.05$ de significancia. Donde podemos observar que entre bloques para todas las variables en evaluación no existe diferencia significativa. Entre tratamientos si existe diferencia significativa para PG, AIM, DM, PS, NP/ML, NLM y LM. Existe diferencia significativa entre densidad para AP, DM, NLM y LM. Entre las variedades existe diferencia significativa para $P G$, AIM, PS, NP/ML y LM. Y entre la interacción existe diferencia significativa para DM, NP/ML y LM. 


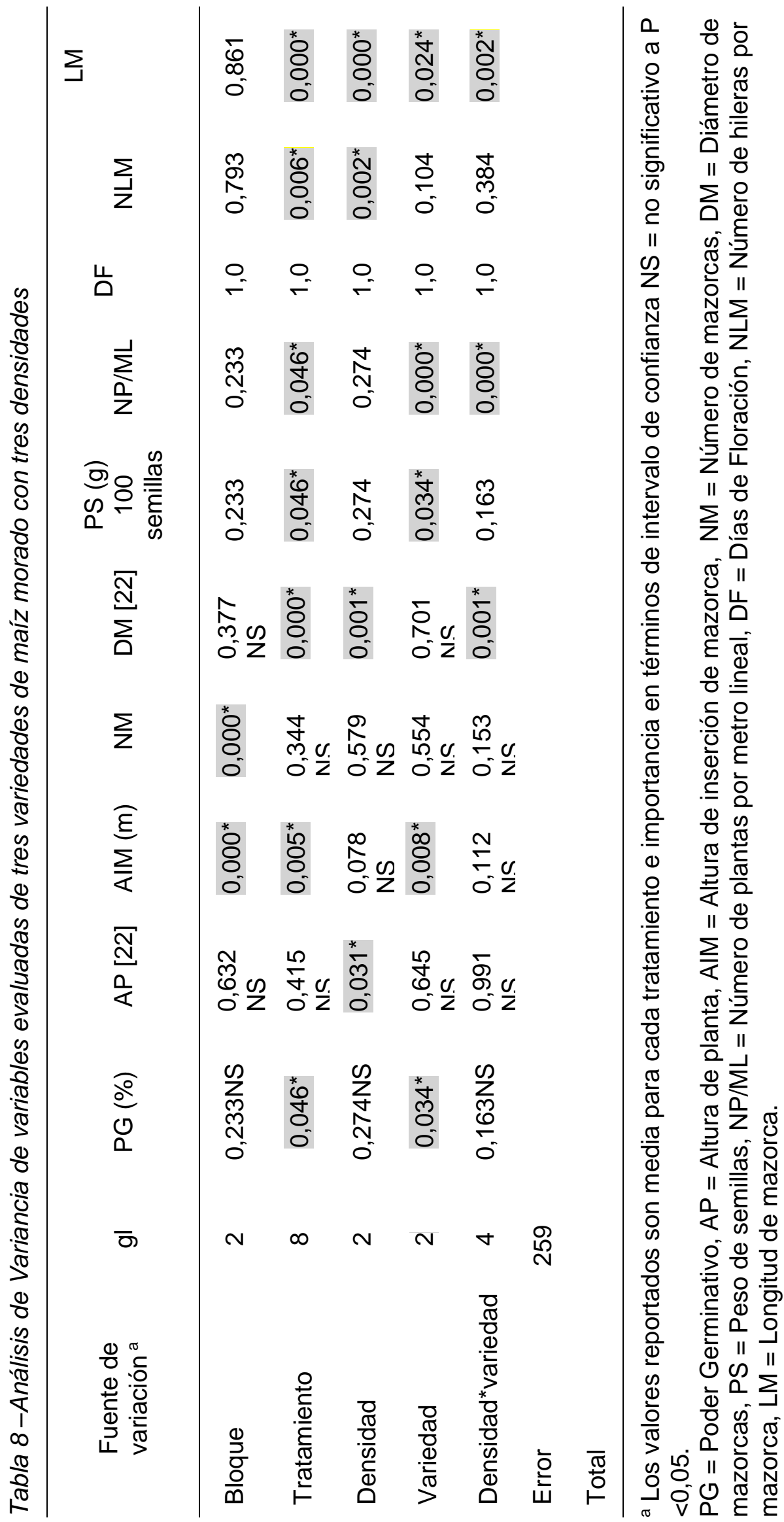


Figura[m5] 2: Porcentaje de germinación (a), altura de planta (b), número de plantas por metro lineal (c), días de floración $(d)$, inserción de mazorca (e), número de mazorca por plantas (f), en función de diferentes tratamientos, (variedades PVM - 581, INIA - 601, UNC - 47 y Densidades: 2.5, 1.5 y $1.2 \mathrm{~m}^{2}$ )

a. Porcentaje de germinación (PG)

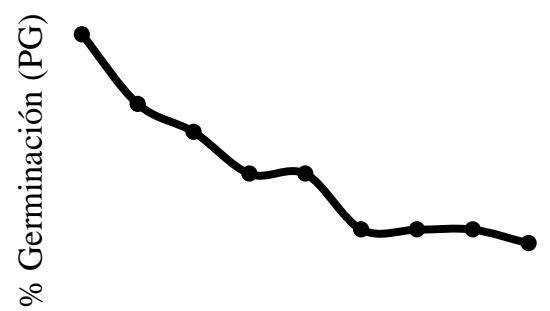

Tratamientos

c. Número de plantas NP/ML

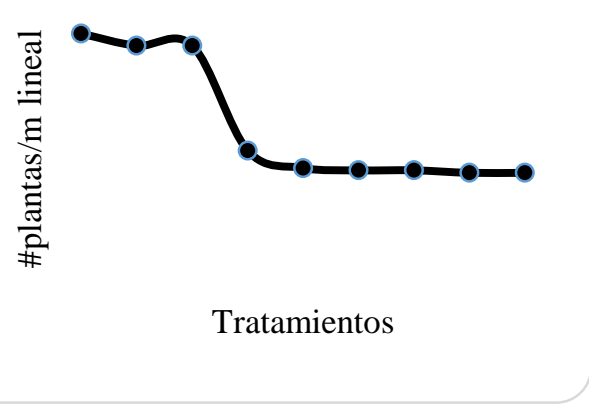

e. Altura de Inserción de mazorca [7]

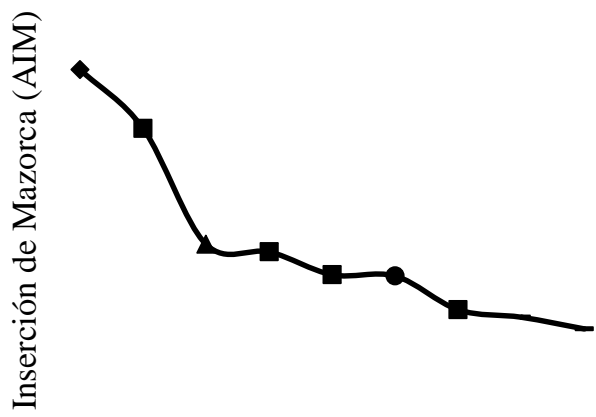

Tratamientos

Fuente: Elaboración propia (referente a la tabla 7 y 8)

De la figura 2,

a. Como se puede observar, el mayor porcentaje de germinación se encuentra en el tratamiento 5 (96.80), el tratamiento 6 y 9 no fueron diferentes mostrando menores porcentajes de germinación. Es b. Altura de planta (AP)

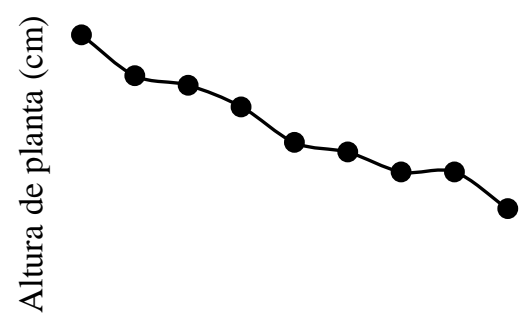

Tratamientos

d. Días de floración (DF)

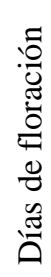

Tratamientos

f. Número de Mazorcas por planta (NM)

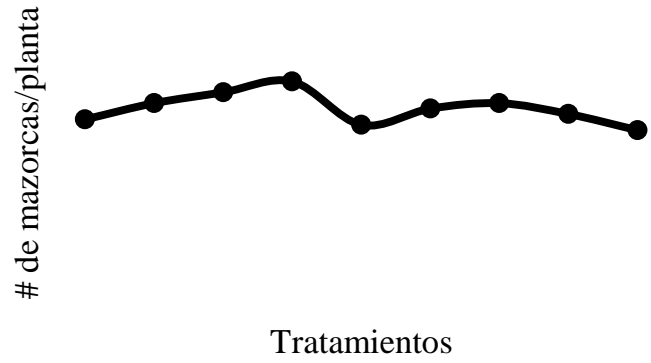

decir la variedad de maíz morado UNC - 47, se corresponde a la densidad $2\left(1.5 \mathrm{~m}^{2}\right)$.

b. Como se puede observar, la mayor altura de planta fue 202.6 y se encuentra en el tratamiento 1 , 
es decir la variedad de maíz morado INIA - 601 (parcela 1), a densidad $60 \mathrm{~cm} \times 40\left(2.4 \mathrm{~m}^{2}\right)$.

c. El mayor \#plantas $/ m$ lineal fue de 4 y se encuentra en el tratamiento 1 variedad INIA - 601 a densidad $60 \mathrm{~cm} \times 40 \mathrm{~cm}\left(2.4 \mathrm{~m}^{2}\right)$.

d. En cuanto a los días de floración se encontró que a los 93 días florecieron en todos los tratamientos a diferentes densidades, es decir, no existe diferencia significativa entre diversos tratamientos. e. Con relación a la inserción de mazorca se observa que la mayor altura de inserción de mazorca se encontró en el tratamiento 9 (1.446 m), es decir, la variedad de maíz PVM - 581 a densidad de $1.20 \mathrm{~m}^{2}$. En cuanto a los menores valores se encuentran en el tratamiento 8 (UNC - 47).

f. En cuanto al número de mazorca por planta el tratamiento $4 \quad(1.43$ mazorcas/planta $\sim 1$ mazorca/planta) es decir la variedad INIA - 601 a densidad $50 \mathrm{~cm} \times 30\left(1.5 \mathrm{~m}^{2}\right)$ y para los demás tratamientos sucede lo mismo.

Figura[m6] 3: numero de hilera por mazorca (g), diámetro de mazorca (h), longitud de mazorca (i) y Peso de semillas (j) en función de diferentes tratamientos (variedades PVM - 581, INIA - 601, UNC - 47 y Densidades: 2.5, 1.5 y $\left.1.2 \mathrm{~m}^{2}\right)$

g. Número de hilera por mazorca (NLM)
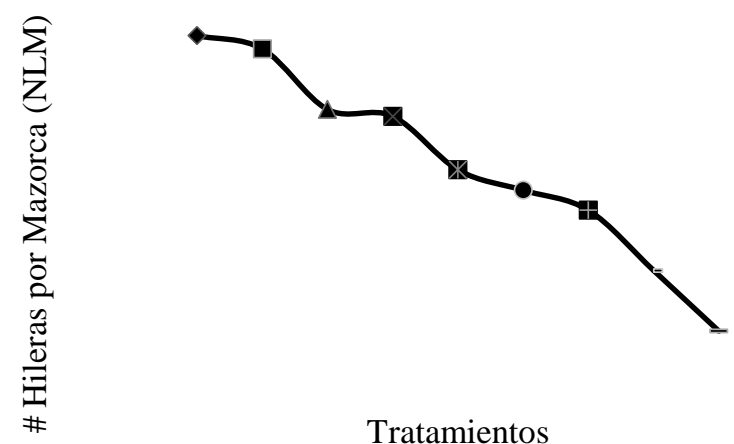

Tratamientos

i. Longitud de mazorca (LM)

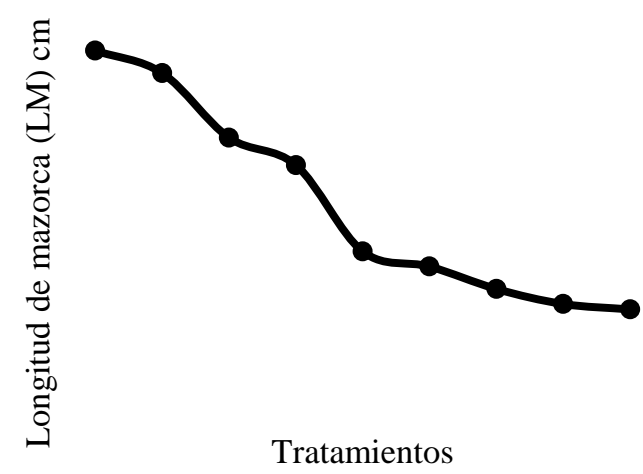

Fuente: Elaboración propia (referente tabla 7 y 8)

De la figura 3

g. El mayor número de hileras por mazorca se encontró en el tratamiento 3 (11.83 \#de hileras por mazorca), es decir, el maíz de la variedad PVM 581 a densidad de $60 \mathrm{~cm} \times 40 \mathrm{~cm}\left(2.4 \mathrm{~m}^{2}\right)$. El menor h. Diámetro de la mazorca (DM) cm

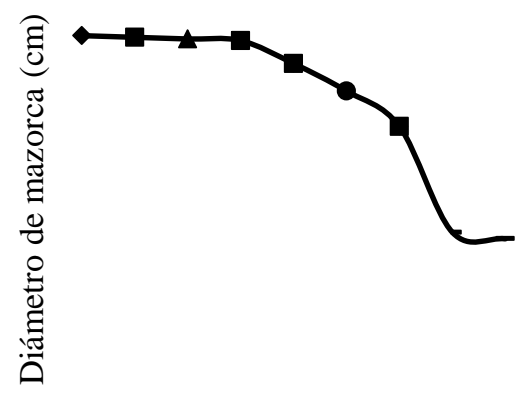

Tratamientos j. Peso de semillas (PS) g

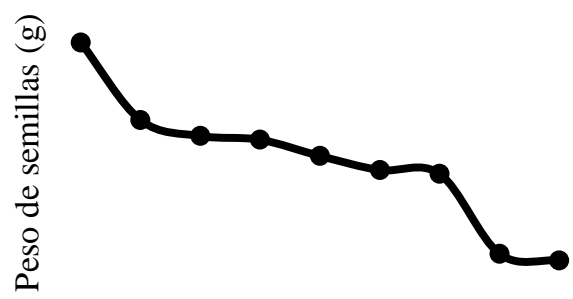

Tratamientos

valor se encontró en el tratamiento 7 de la misma variedad a densidad de $1.20 \mathrm{~m}^{2}$. 
h. El mayor diámetro de mazorca se encuentra en el tratamiento 6 (4.93), es decir, la variedad PVM 581 a densidad de $1.5 \mathrm{~m}^{2}$ seguido por el tratamiento 8 , 1 y 3 con poca diferencia (4.927). El menor diámetro de mazorca se encuentra en el trat. 7 y 9 , es decir, la misma variedad a densidad de $1.2 \mathrm{~m}^{2}$.

i. La mayor longitud de mazorca se encontró en el tratamiento $3(20.76 \mathrm{~cm})$, es decir, en la variedad PVM - 581 a densidad de $2.4 \mathrm{~m}^{2}$. La menor longitud se encuentra en el mismo tratamiento, pero a densidad de $1.2 \mathrm{~m}^{2}$.

j. El mayor peso de semillas se encontró en el tratamiento 4 (5.497), es decir, en la variedad INIA - 601 a densidad de $1.5 \mathrm{~m}^{2}$. El menor peso de semillas se encontró en el tratamiento 2 , variedad UNC - 47 a densidad de $2.4 \mathrm{~m}^{2}$.

Figura[M7] 4 - Longitud promedio de mazorcas según densidad y variedad de maíz

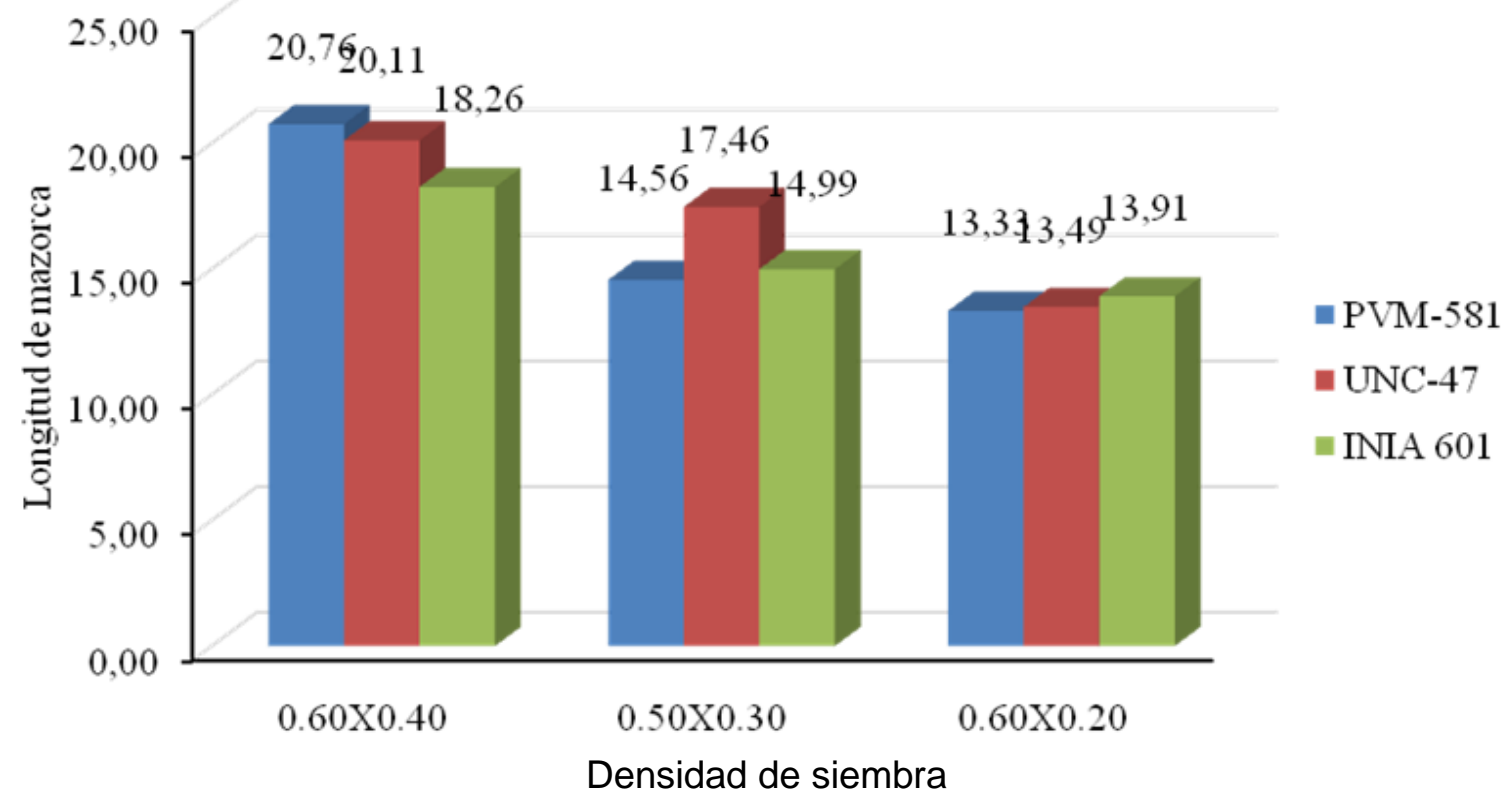

Con respecto a la comparación entre las tres densidades y tres variedades de maíz; la longitud promedio de mazorca fue la variedad PVM - 581 a densidad $2.4 \mathrm{~m}^{2}$. La variedad UNC -47 a densidad de siembra de $1.5 \mathrm{~m}^{2}$. A densidades de $1.2 \mathrm{~m}^{2}$ la que obtuvo mayor tamaño de mazorca fue la variedad INIA - 601 .

\subsection{Resultados de Rendimiento}

Tabla 9-Comparación media del híbrido, rendimiento de densidad y rendimiento del maíz

\begin{tabular}{|c|c|c|c|c|c|c|}
\hline \multirow{2}{*}{\multicolumn{2}{|c|}{ TRATAMIENTO }} & \multirow{2}{*}{ VARIEDAD*DENSIDAD } & \multirow{2}{*}{$\begin{array}{c}\text { Rendimiento } \\
\text { promedio } \\
\mathrm{Kg} / \mathrm{Ha}\end{array}$} & \multirow{2}{*}{$\begin{array}{c}\text { Error } \\
\text { estándar }\end{array}$} & \multicolumn{2}{|c|}{$\begin{array}{c}\text { Intervalo de confianza al } \\
95 \% \\
\end{array}$} \\
\hline & & & & & $\begin{array}{l}\text { Límite } \\
\text { inferior }\end{array}$ & $\begin{array}{l}\text { Límite } \\
\text { superior }\end{array}$ \\
\hline & 1 & INIA 601-0.60X0.40 & 4808.80 & 29.493 & 4750.723 & 4866.877 \\
\hline 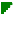 & 2 & INIA 601-0.50X0.30 & 4295.70 & 29.493 & 4237.623 & 4353.777 \\
\hline 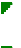 & 3 & INIA 601-0.60X0.20 & 4114.67 & 29.493 & 4056.590 & 4172.744 \\
\hline 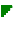 & 4 & UNC $47-0.60 \times 0.40$ & 3974.13 & 29.493 & 3916.056 & 4032.210 \\
\hline 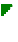 & 5 & UNC 471-0.50X0.30 & 3661.67 & 29.493 & 3603.590 & 3719.744 \\
\hline 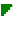 & 6 & UNC 47-0.60X0.20 & 3379.73 & 29.493 & 3321.656 & 3437.810 \\
\hline 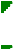 & 7 & PVM 581-0.60X0.40 & 2921.60 & 29.493 & 2863.523 & 2979.677 \\
\hline & 8 & PVM 581-0.50X0.30 & 2817. 10 & 29.493 & 2759.023 & 2875.177 \\
\hline 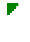 & 9 & PVM 581-0.60X0.20 & 2745.50 & 29.493 & 2687.423 & 2803.577 \\
\hline
\end{tabular}




\section{Discusión}

La variedad de maíz morado UNC - 47 obtuvo un porcentaje de germinación de 96.80, se corresponde a la densidad $2\left(1.5 \mathrm{~m}^{2}\right)$ trat. 5, posiblemente por la variación de dosis aplicada de fertilizante según sus requerimientos a pesar que se usó la menor cantidad de $\mathrm{N}-\mathrm{P}_{2} \mathrm{O} 5-\mathrm{K}_{2} \mathrm{O} \mathrm{kg} / \mathrm{ha}$ (160 - 140 - 120), además, menor fertilización de mezcla de urea + fosfato diamónico + cloruro de potasio g/planta (5.81) y menor en $1 \mathrm{~g} /$ planta es decir $4.64 \mathrm{~g}$.

La mayor altura de planta fue $202.6 \mathrm{~cm}$ (trat. 1), es decir, variedad de maíz morado INIA - 601 (parcela 1 ), a densidad $60 \mathrm{~cm} \times 40\left(2.4 \mathrm{~m}^{2}\right)$. Valores cercanos se encontraron en variedad INIA - 601 de $2.16 \mathrm{~m}$ $216 \mathrm{~cm}$ indicado por INIA (2014), posiblemente por las densidades entre plantas y surcos, la dosis de fertilización, el fraccionamiento de aplicación de nitrógeno.

El mayor número de plantas/m lineal fue de 4 (trat. 1) variedad INIA - 601 a densidad $60 \mathrm{~cm} \times 40 \mathrm{~cm}(2.4$ $\mathrm{m}^{2}$ ). Tiene relación a la altura de plantas aunque difiere en el distanciamiento de siembra sugerido por Lazo (1999) [43], que se verá reflejado en el rendimiento, así como, la fertilización de acuerdo al requerimiento del suelo (INIA, 2014).

Los días de floración para todas las variedades ocurrieron a los 93 días. Valores cercanos a la variedad INIA - 601 encontrados a los 98 días (INIA, 2014); para siembra en sierra media ocurre entre los 90 y 105 días [42], posiblemente porque esta variedad tolera alturas de 2600 y 2900 msnm. A diferencia a los valores de la variedad Caraz PVM 581 , sembrado a alturas de 1,200-4,000 msnm es decir en costa central y sierra media [43] su floración femenina ocurre de 55 a 60 días después de la siembra [42]. Para la altura de inserción de mazorca la variedad PVM - 581, fue $145 \mathrm{~cm}$ superior a las otras variedades. Es decir, a densidad de $1.20 \mathrm{~m}^{2}$ $(60 \mathrm{~cm} \times 20 \mathrm{~cm})$.

En cuanto a los menores valores se encuentran en el tratamiento 8 (UNC - 47). Valores cercanos de $144 \mathrm{~cm}$ encontrados por Garay et al. (2014) [41].

El número de mazorca/planta (1.43 mazorcas/planta 1 mazorca/planta) la variedad INIA - 601 a densidad $50 \mathrm{~cm} \times 30\left(1.5 \mathrm{~m}^{2}\right)$ donde las otras medias del experimento (1.13 a 1.20 mazorcas) son próximos al rango 1.0 - 1.3 evaluados en genotipos de maíz dulce [41]; asimismo, datos cercanos a los valores encontrados por INIA, 2014 de 1 a 2 mazorcas/planta.

El mayor número de hileras por mazorca se encontró en la variedad PVM - 581 (11.83 \#de hileras por mazorca) a densidad de $2.4 \mathrm{~m}^{2}$. Valores encontrados por encima de [41] entre 11 y 17 hileras. En la variedad INIA - 601 entre 10 a 12 (INIA, 2014). Los valores van a variar depende a la densidad de siembra, variedad sembrada.

El mayor diámetro de mazorca fue en la variedad PVM - $581(4.93 \mathrm{~cm})$ a densidad de $1.5 \mathrm{~m}^{2}$; cercano a los valores $(4.6 \mathrm{~cm})$ de la variedad INIA - 601 (INIA, 2014) y los encontrados por Garay, Brizuela, \& Oviedo, 2014) $4.81 \mathrm{~cm}$. Además de los resultados no se apreció diferencia significativa entre las variedades INIA - 601 y UNC - 47 a densidades de 2.4 y $1.2 \mathrm{~m}^{2}$ respectivamente. El menor diámetro de mazorca está a densidad de $1.2 \mathrm{~m}^{2}$ de la misma variedad.

La mayor longitud de mazorca está en la variedad PVM - $581(20.76 \mathrm{~cm})$ a densidad de $2.4 \mathrm{~m}^{2}$. La menor longitud es de $13.33 \mathrm{~cm}$ de la misma variedad a densidad de $1.2 \mathrm{~m}^{2}$. De acuerdo a registros de INIA - 601 es de $17.5 \mathrm{~cm}$; y valores por Garay et al. (2014) [41] fueron entre 17.70 y 18.85 $\mathrm{cm}$.

El mayor peso de semillas $5.497 \mathrm{~g}$, se encontró en la variedad INIA - 601 a densidad de $1.5 \mathrm{~m}^{2}$ trat. 4 . El menor peso de semillas se encontró en la variedad UNC - 47 a densidad de $2.4 \mathrm{~m}^{2}$.

$456,2 \mathrm{~g} / 1000$ semillas $=($ INIA, 2014 $)$

$3901.5 \mathrm{~g} / 1000$ semillas $=39.015 \mathrm{~g} / 1000$ semillas

$(265,25 \mathrm{~g}) / 1000$ semillas [41]

$5.497 \mathrm{~g}=549.7$ peso en 1000 semillas.

El rendimiento promedio de maíz morado fue de $4808.80 \mathrm{~kg} / \mathrm{ha}$ y se encontró en la variedad INIA 601 a densidad $60 \mathrm{~cm} \times 40\left(2.4 \mathrm{~m}^{2}\right)$ y por consiguiente menor diámetro de mazorca. Valores de rendimiento que difieren por los encontrados por Yánez et al. $(-2016)$ [2] de 3.05 TM/ha. De acuerdo con Parihar et al. (2016) [22] el rendimiento del maíz se ve influenciado por las condiciones climáticas, variedad o genotipo y objetivo de estudio. Menores rendimiento se encontraron PVM - 581 a densidades $2745.50 \mathrm{~kg} / \mathrm{ha}$ el bajo rendimiento puede deberse al estrés hídrico [25]. Asimismo $_{2}$ la densidad de siembra dependerá del clima y las variedades. En el caso de híbridos se utiliza de 15 a $20 \mathrm{~kg} / \mathrm{ha}$ y se siembra a una distancia de 75 a $100 \mathrm{~cm}$ entre surco y de 20 a $25 \mathrm{~cm}$ entre plantas. La semilla debe ser de calidad para garantizar una óptima producción. De ello, se puede determinar que, las variedades en estudios fueron tolerantes a las condiciones climáticas. Hubo poca presencia de roja en la hoja (Puccinia sorghi) pero sí se presentó en las mazorcas granos flojos en poca cantidad. 
En el 2006, el rendimiento nacional de maíz morado con variedades de este estudio, logrado con tecnología media, fue de $4.675 \mathrm{~kg} / \mathrm{ha}$. Este valor es $3,7 \%$ mayor que el rendimiento registrado en el 2005. La región de Cajamarca presenta el mayor rendimiento a nivel nacional, con $8,389 \mathrm{~kg} / \mathrm{ha}$, estándar superior al promedio nacional. Apurímac y Huánuco también tienen rendimientos altos en este cultivo, llegando en el 2006 a $8_{-1} 1$ y $7_{-9}^{-1} 1 \mathrm{Tm} / \mathrm{ha}$. El rendimiento de maíz morado logrado en Ayacucho es bajo (3.99 Tm/ha) y es inferior al promedio nacional. En el ranking nacional, los menores resultados de rendimiento se obtienen en las regiones de Ayacucho Moquegua, Lambayeque y Lima.

La producción de maíz comercial en el Perú según la FAO, en el año 2013 alcanzó una producción de 32777.0 (32 mil toneladas/hectárea), lejos de alcanzar con variedades nativas de maíz morado.

Una comprensión clara de la relación densidad de siembra - comportamiento agronómico, es tener en cuenta diversos factores importantes, que se muestran en las curvas de rendimiento en producción de maíz morado. Sin embargo, los avances en las técnicas de manejo de cultivos, tales como, un mayor uso de fertilizantes inorgánicos, área de riego, técnicas de malezas y control de plagas mejoradas también fueron factores críticos para el aumento tanto de la densidad de siembra y rendimiento. Es necesario práacticas de siembra en surcos con distancias entre ellos por $70 \mathrm{~cm}$, usando 2 a 3 semillas por golpe y con una distancia de 40 a $50 \mathrm{~cm}$ por golpe, del mismo modo, de acuerdo a la calidad de los suelos y su nivel de fertilidad, es entre 55.56 y 66.67 plantas/ha [42].

\section{Conclusiones}

Se evaluó el efecto de tres densidades de siembra en el comportamiento agronómico en tres variedades de maíz morado (Zea mayz L.) INIA $601, \mathrm{UNC}-47$ y PMV - 581.

Se identificó las mejores densidades y variedad así como los parámetros agronómicos. Para el análisis de varianza estadística se usó la prueba Tukey, en el programa SPSS Versión 23.0.

El mayor \% de germinación de 96.80 fue para la variedad de maíz morado UNC - 47 a densidad de siembra de $1.5 \mathrm{~m}^{2}$. Los días de floración fueron a los 93 días sin diferencia significativa entre los demás tratamientos.

La variedad INIA - 601 sobresalió en mayor altura de planta fue $202.6 \mathrm{~cm}$ (trat. 1) a densidad $60 \mathrm{~cm} \times 40$ $\left(2.4 \mathrm{~m}^{2}\right)$. Asimismo $_{2}$ para \#plantas $/ \mathrm{m}$ lineal (4), número de mazorca/planta (1.43) a densidad $50 \mathrm{~cm} \times 30\left(1.5 \mathrm{~m}^{2}\right)$ y mayor peso de semillas $5.497 \mathrm{~g}$.
En cambio la variedad PVM - 581 sobresalió para altura de inserción de mazorca fue $145 \mathrm{~cm}$ superior a las otras variedades, es decir, a densidad de 1.20 $\mathrm{m}^{2}(60 \mathrm{~cm} \times 20 \mathrm{~cm}$ ); así como para mayor número de hileras por mazorca (11.83 \#de hileras por mazorca) y mayor longitud de mazorca $(20.76 \mathrm{~cm})$ a densidad de $2.4 \mathrm{~m}^{2}$. La menor longitud fue de $13.33 \mathrm{~cm}$ de la misma variedad a densidad de 1.2 $\mathrm{m}^{2}$. El mayor diámetro de mazorca está en la variedad PVM - $581(4.93 \mathrm{~cm})$ a densidad de 1.5 $\mathrm{m}^{2}$. Además de los resultados no se apreció diferencia significativa entre las variedades INIA 601 y UNC - 47 a densidades de 2.4 y $1.2 \mathrm{~m}^{2}$ respectivamente. El menor diámetro de mazorca está a densidad de $1.2 \mathrm{~m}^{2}$ de la misma variedad.

El rendimiento promedio de maíz morado fue de $4808.80 \mathrm{~kg} / \mathrm{ha}$ y se encontró en la variedad INIA 601 a densidad $60 \mathrm{~cm} \times 40\left(2.4 \mathrm{~m}^{2}\right)$ y por consiguiente menor diámetro de mazorca. Menores rendimiento se encontraron PVM - 581 a densidades 2745.50 $\mathrm{kg} / \mathrm{ha}$.

\section{Recomendaciones}

Realizar la siembra en estación de menor lluvia ya que al fertilizar, estos elementos pueden ser arrastrados antes de que sean disponibles para la planta y ocurran pérdidas por lixiviación, escorrentía y/o evaporación. Es recomendable mantener la humedad del suelo con buena aireación, evitar que la luz llegue a las raíces y proteger del ataque de plagas y enfermedades.

Realizar siembra a surco corrido, 2 semillas cada 15 $\mathrm{cm}$ con densidad 82,00 plantas/ha (para los dos distanciamientos). Densidades mayores pueden producir rendimientos más elevados, teniendo en cuenta el manejo del cultivo, pero se corre el riesgo de obtener mucha plantas improductivas y mazorcas más pequeñas, con menor tamaño de grano, afectando el rendimiento. Realizar análisis físico - químico con la finalidad de determinar la cantidad de antioxidantes que tiene las variedades en estudio. Buscar alternativa para dar valor agregado a la producción de maíz morado. Realizar estudios se siembra de maíz morado, también de otras especies en un largo plazo no menor de 5 años, para ver la adaptación de la especie de maíz morado, obviamente haciendo una rotación de cultivo para evitar plagas y enfermedades que afectan los cultivos. Investigar las necesidades nutricionales en las plantas de maíz morado con altas densidades de siembra, en función del número de plantas. Capacitar a los agricultores sobre la siembra de diferentes variedades de maíz morado que se puedan adaptar en la región Amazonas, así 
aumentaría el crecimiento sostenido y económicoz de la población rural, contribuyendo a la seguridad alimentaria y nutricional.

\section{Agradecimientos}

Al Instituto de Investigación para el Desarrollo Sustentable de Ceja de Selva (INDES-CES), al Ing. Manuel Oliva coordinador del proyecto "Creación del servicio de Laboratorio de Biotecnología y Fisiología Vegetal", a los colaboradores, al Laboratorio de Investigación en Suelos y Aguas (LABISAG).

\section{Referencias}

[1] Tajul, M., et al., Influence of plant population and nitrogen-fertilizer at various levels on growth and growth efficiency of maize. The Scientific World Journal, 2013. 2013.

[2] Yánez, G., et al., Ficha técnica de la variedad de Maíz Negro INIAP-199 "Racimo de Uva". 2016.

[3] González, S.L., G.G. Bazán, and L.C. Chávez, Zea mays L."maíz morado"(Poaceae), un cereal utilizado como alimento en el Perú prehispánico. ARNALDOA, 2016. 23(1): p. 295-316.

[4] Cobo, B., Historia del nuevo mundo. Vol. 1. 1890: Imp. de E. Rasco.

[5] Sauñi, P. and H. Fernando, Utilización de la antocianina del maíz morado (Zea mays L.) y stevia (Stevia rebaudiana bertoni) en la elaboración de un producto tipo mermelada y su aceptabilidad. 2014.

[6] Fredy, Q.J., Características morfológicas y químicas de 3 cultivares de maíz morado (Zea mays L.) en Arequipa-Perú. Revista de la Sociedad Química del Perú, 2016. 77(3).

[7] Ramírez Prado, F. and J. Deza Rivasplata, Cuando los desiertos eran bosques. 2000, Fondo Editorial de la Universidad Alas Peruanas.

[8] Molina, M.J.C. and L. Córdova, Informe Nacional sobre el estado de los Recursos Fitogenéticos para la Agricultura y la Alimentación. Recuperado el día, 2006. 14.

[9] Yang, Z., et al., Extraction and identification of anthocyanin from purple corn (Zea mays L.). International Journal of Food Science and Technology, 2009. 44(12): p. 2485-2492.

[10] Quispe Jacobo, f. and k. Arroyo Condorena, Gorriti Gutierrez, caractristicas morfologicas y quimicas de 3 cultivares de maiz morado. revista de la sociedad quimica del peru, 2011: p. 205-217.

[11] Somavat, P., et al., Coproduct yield comparisons of purple, blue and yellow dent corn for various milling processes. Industrial Crops and Products, 2016. 87: p. 266-272.

[12] Gamarra, F.M.C., et al., Extraction of corn colorants (Zea mays L.). Ciencia e Tecnologia de Alimentos, 2009. 29(1): p. 62-69.

[13] Jing, P. and M. Giusti, Effects of Extraction Conditions on Improving the Yield and Quality of an Anthocyanin- Rich Purple Corn (Zea mays L.) Color Extract. Journal of food science, 2007. 72(7): p. C363-C368.

[14] Brag Egg, A., Diccionario enciclopédico de plantas útiles del Perú. Programa de las Naciones Unidas para el Desarrollo, Lima (Peru) 1999.

[15] Pedreschi, R. and L. Cisneros-Zevallos, Phenolic profiles of Andean purple corn (Zea mays L.). Food Chemistry, 2007. 100(3): p. 956-963.

[16] Yang, Z., et al., Thermal degradation kinetics of aqueous anthocyanins and visual color of purple corn (Zea mays L.) cob. Innovative Food Science \& Emerging Technologies, 2008. 9(3): p. 341-347.

[17] Yang, Z., et al., Optimization extraction of anthocyanins from purple corn (Zea mays L.) cob using tristimulus colorimetry. European Food Research and Technology, 2007. 227(2): p. 409-415.

[18] Castañeda, B.C., L.A. Ibañez, and R.M. Mejía, Estudio fitoquímico y farmacológico del Zea mays L. amilaceae st (maíz morado).

[19] Molina, M. and L. Córdova, Informe Nacional sobre el estado de los Recursos Fitogenéticos para la Agricultura y la Alimentación. Recuperado el día, 2006. 14.

[20] Fries, A.M. and M.E. Tapia, Guía de campo de los cultivos andinos. 2007: FAO, ANPEPERÚ.

[21] Yánez, C., et al., Inventario Tecnológico del Programa de Maíz. INIAP, Quito, Ecuador, 2005: p. 2-25.

[22] Parihar, C., et al., Conservation agriculture in irrigated intensive maize-based systems of north-western India: Effects on crop yields, water productivity and economic profitability. Field Crops Research, 2016. 193: p. 104-116. 
[23] Tapia, M., et al., Agronomía de los cultivos andinos. Cultivos andinos subexplotados. FAO. Santiago de Chile, 2001.

[24] Silva, D.V., et al., Crescimento e rendimento do milho sob interferência da tiririca. Semina: Ciências Agrárias, 2015. 36(5): p. 3077-3084.

[25] Ruane, A.C., et al., Climate change impact uncertainties for maize in Panama: Farm information, climate projections, and yield sensitivities. Agricultural and Forest Meteorology, 2013. 170: p. 132-145.

[26] González, S., Córdova, H., Rodríguez, S., De León, H., \& Serrato, V. M., Determinacion de un patron heterotico a partir de la evaluacion de un dialelo de diez lineas de maiz subtropical. Agron Mesouam, 1997: p. 1-7.

[27] Sangoi, L., Understanding plant density effects on maize growth and development: an important issue to maximize grain yield. Ciência Rural, 2001. 31(1): p. 159-168.

[28] NiK, M.M., et al., Effect of plant density on yield and yield components of corn hybrids (Zea mays). Scientific Research and Essays, 2011. 6(22): p. 4821-4825.

[29] Lindsey, A.J. and P.R. Thomison, DroughtTolerant Corn Hybrid and Relative Maturity Yield Response to Plant Population and Planting Date. Agronomy Journal, 2016. 108(1): p. 229-242.

[30] KEATING, B.A., B. Wafula, and R.M. COWN, Simulation of Plant Density Effects on Maize Yield as Influenced by Water and Nitrogen Limitations. 1988.

[31] Assefa, Y., et al., Yield Responses to Planting Density for US Modern Corn Hybrids: A Synthesis-Analysis. Crop Science, 2016. 56(5): p. 2802-2817.

[32] Abbas, H.K., et al., Effect of planting density, irrigation regimes, and maize hybrids with varying ear size on yield, and aflatoxin and fumonisin contamination levels. American Journal of Plant Sciences, 2012. 3(10): p. 1341.

[33] Sun, S., et al., Effects of planting density on soil moisture and corn yield under plastic film mulching in a rain-fed region of northeast China. Chinese J Ecol, 2014. 33: p. 26502655.

[34] Woli, P., et al., Predicting crop yields with the agricultural reference index for drought. Journal of agronomy and crop science, 2014. 200(3): p. 163-171.
[35] Tokatlidis, I.S., Adapting maize crop to climate change. Agronomy for Sustainable Development, 2013. 33(1): p. 63-79.

[36] Bonilla, N., Manual de recomendaciones técnicas del cultivo de maíz. Instituto Nacional de Innovación y transferencia en tecnología Agropecuaria (INTA). San José-Costa Rica. p, 2009. 17.

[37] Manrique, A., El maíz en el Perú. CONCYTEC. Serie tecnológica, 1997(10).

[38] Guillén-Sánchez, J., Mori-Arismendi, S., \& Paucar-Menacho, L. M., Characteristics and functional properties of purple corn (Zea mays L.) Scientia Agropecuaria, 2014: p. 211-217.

[39] Solís, J.D.Á., et al., Balance parcial de nitrógeno en el sistema de cultivo de maíz (Zea mays L.) con cobertura de leguminosas en Chiapas, México. Agronomía Costarricense, 2016. 40(1).

[40] Bayer, C. and J. Mielniczuk, Características químicas do solo afetadas por métodos de preparo e sistemas de cultura. Revista brasileira de Ciência do Solo, 1997. 21(1): p. 105-112.

[41] Garay, C.R.E., J.M.M. Brizuela, and V.S. Oviedo, Evaluación de genotipos de maíz dulce. Investigación Agraria, 2014. 14(2): p. 81-86.

[42] Florencio Requis Varillas (2012). Manejo agronómico del maíz morado en los valles interandinos del Perú.

[43] Lazo Cama Rafael Néstor. Fertilización potásica y fosfórica en el rendimiento de maíz morado (Zea mays L.) P.M. 581, El Cural Arequipa. Escuela Profesional de Agronomía, UNSA, Arequipa. 146 pp. (1999).

[44] Zevallos, M. Efectos del Nitrógeno y el Ergostin en la producción de Maíz Morado (Zeamaydis L.) en Sierra Media. UNSA. Arequipa-Perú. (1999)

[45] Universidad Nacional de Cajamarca. PUBLICADO EL 12 - MAR - 2013. gaceta universitaria/UNC

[46] CENTRUM, Centro de Negocios Pontificia Universidad Católica del Perú (2007) Mercado del Maíz Morado_Panorama Actual y Perspectivas de Exportación, Solid Perú.

[47] Registros de producción por provincias (2007). Oficina de Información Agraria de la DRA. Ayacucho. 